\title{
Thermo-mechanical coupling of a viscoelastic-viscoplastic model for thermoplastic polymers: thermodynamical derivation and experimental assessment
}

\author{
A. Krairia,*, I. Doghri ${ }^{\text {b }}$, J. Schalnat ${ }^{a}$, G. Robert ${ }^{c}$, W. Van Paepegem ${ }^{a}$ \\ ${ }^{a}$ Department of Materials, Textiles and Chemical Engineering (MaTCh), Ghent University, \\ Technologiepark 903, 9052 Zwijnaarde, Belgium \\ ${ }^{b}$ Université catholique de Louvain (UCL), IMMC, Bâtiment Euler, 4 Avenue G.Lemaître \\ B-1348 Louvain-La-Neuve, Belgium \\ ${ }^{c}$ Solvay Performance Polyamides, Avenue Ramboz, 69190 Saint-Fons, France
}

\begin{abstract}
In this paper, a new constitutive model is proposed for the behavior of thermoplastic polymers under non-isothermal conditions. The model couples linear viscoelasticity and viscoplasticity and thermal effects. It is formulated within the framework of irreversible thermodynamics. The total strain is the sum of viscoelastic, viscoplastic and thermal strains. General hereditary integrals describe the thermo-viscoelastic response. The viscoplastic part accounts for both isotropic and kinematic hardenings. The stress-strain response and the material self-heating are predicted and compared to experimental data on Polyamide 66 (PA66) and Polypropylene (PP). Good agreement between the numerical simulations and experimental data was obtained for the two materials.
\end{abstract}

Keywords: Polymeric material, constitutive behavior, rate-dependent material, thermomechanical processes, self-heating

\section{Introduction}

As their domain of application is in continuous expansion, thermoplastic polymers especially, semi-crystalline ones, are being exposed to more and more

4 challenging working conditions with complex thermo-mechanical loading. In particular, the variation of temperature and loading rate have important effects

\footnotetext{
* Corresponding author

Email addresses: anouar.krairi@ugent.be (A. Krairi), issam.doghri@uclouvain.be (I. Doghri), Joanna.Schalnat@ugent.be (J. Schalnat), gilles.robert@solvay.com (G. Robert), wim.vanpaepegem@ugent.be (W. Van Paepegem)
} 
6 on the material response (Zhou and Mallick, 2002; Dasari and Misra, 2003; Krempl and Khan, 2003; Khan and Farrokh, 2006; Farrokh and Khan, 2010; Reis

$8 \quad$ et al., 2013). Crystalline and amorphous phases of the material are responsible for its complex behavior, which is characterized by reversible and irreversible deformations (Kennedy et al., 1994; Ayoub et al., 2011).

Different approaches were proposed to model semi-crystalline polymers. These 12 approaches can be classified in two classes: the first class is physical models inspired from the material micro-structure characterized by crystalline and amorphous phases. The second class is phenomenological models that treat the material as an homogeneous medium which exhibits reversible and/or irreversible 16 deformations.

The first class of models is motivated by the difference in response of each 18 phase of the material: amorphous and crystalline phases. Some authors believe that the crystalline phase has the most important contribution to the material behavior, especially at small deformation conditions and the amorphous phase is rather important at large deformation stage, or more specifically in the post22 yielding regime of the material response (Garcia-Gonzalez et al., 2017). In order to take into account the effect of the material crystallization degree, multi-scale

24 approaches were employed in several works such as (Nikolov and Doghri, 2000; Nikolov et al., 2002; Van Dommelen et al., 2003; Makradi et al., 2005; Bedoui 26 et al., 2006; Gueguen et al., 2008; Ayoub et al., 2011; Uchida and Tada, 2013; Alisafaei et al., 2016).

28 On the other hand, those who believe that the amorphous phase has the most important contribution and focus on the study of polymers with large deforso mations, were able to benefit from the important amount of work dedicated to the pure amorphous polymers (see for review Bouvard et al. (2009)). This work started since the middle of the twentieth century and mainly two reference models were extensively used and developed through the years: Edwards 34 and Vilgis (1986) model and Haward and Thackray (1968) model. The model of Edwards and Vilgis (1986) is also famous as the network model, it is based on the work of Ball et al. (1981), that modified the classical rubber elasticity (e.g. hyper-elasticity) by proposing the concept of slip-link to account for the sв entanglement slippage along the network chains. The original work of Edwards 
and Viligis was employed and extended by several authors for the amorphous glassy polymers such as Sweeney and Ward (1995) and Billon (2012). The theory was also applied and extended for semi-crystalline polymers in several 42 works such as Sweeney et al. (2002) and Maurel-Pantel et al. (2015). In the work of Maurel-Pantel et al. (2015), the authors extended the model of Bil-

44 lon (2012) to non-isothermal conditions, The original model was developed for time-dependent mechanical behavior of polymers close to the glass transition.

46 Haward and Thackray (1968) proposed a 1D model for large deformation of polymers below their glass transition temperature. Boyce, Parks and Argon

48 (1988) extended the original model to 3D description famous as BPA model. The model was initially based on the three chain concept, then it was extended so by Arruda et al. (1995) to the eight chain model. Further development based on the original Haward and Thackray model was carried out by several au-

52 thors (e.g. Wu and Van Der Giessen, 1993; Arruda et al., 1995; Govaert et al., 2000), other versions for amorphous polymers were proposed by Buckley and 54 co-workers (e.g. Buckley and Jones, 1995; Li and Buckley, 2009) and, Anand and co-workers (e.g. Anand and Gurtin, 2003; Anand et al., 2009; Ames et al., 56 2009).

The second class consists of phenomenological models, which focus on the re58 versible part of the material behavior or the irreversible one or both of them, as the non-linearity is one of the key features of the material behavior. If the ma6o terial deformation is assumed to be governed by reversible deformations, several authors proposed to model the material as non-linear viscoelastic material (e.g.

62 Lai et al., 2005; Khan et al., 2006), based on the theory proposed by Schapery (1969). Other works focus on the rate dependency of the irreversible behavior by 64 using viscoplastic models (e.g. Bardenhagen et al., 1997; Colak, 2005; Drozdov and Christiansen, 2007; Ghorbel, 2008; Dusunceli and Colak, 2008; Drozdov,

66 2011; Khan and Yeakle, 2011) mainly employing the over-stress (VBO) model. Another set of models couples the viscoelastic (VE) and viscoplastic (VP) be68 haviors (e.g. Hasan and Boyce, 1995; Frank and Brockman, 2001; Miled et al., 2011; Yu et al., 2016; Gudimetla and Doghri, 2017).

7o The progressive material degradation was also modeled by coupling the damage to the elasto-viscoplastic (EVP) behavior (e.g. Zairi et al., 2008; Balieu et al., 
2013) or to the viscoelastic and viscoplastic behavior such as the model proposed by two of the authors Krairi and Doghri (2014) based on the work of Miled 74 et al. (2011). Praud et al. (2017) also proposed a model which couples VE, VP and ductile damage, mainly with different description of the viscoelastic part of the behavior using differential representation instead of integral description in the work of Krairi and Doghri (2014), more differences are given in details 78 in Praud et al. (2017). The later model was described as a multi-mechanisms (MM) constitutive model, since several mechanisms are involved to simulate the overall behavior of the material. Cayzac et al. (2013a) also proposed to model the damage in semi-crystalline polymers using a new version of the MM model 82 of Regrain et al. (2009).

The vast majority of the above listed models are isothermal models. In order s4 to be used under non-isothermal conditions, their material parameters need to be calibrated for different temperatures. This method is only valid under a s6 field of temperature with constant magnitude. However, for coupled thermomechanical analysis with variable temperature field, non-isothermal models are s8 more suitable such as the hyperelastic-thermoviscoplastic constitutive model proposed by Garcia-Gonzalez et al. (2017).

90

In this work, based on an extension of the model published by Miled et al. (2011), a new model is proposed for thermoplastic polymers within the frame92 work of irreversible thermodynamics. It couples viscoelasticity and viscoplasticity under non-isothermal loading conditions.

94

The paper is organized as follows. Section 2 presents detailed development of the constitutive model based on a thermodynamics framework. In section 3 , numerical simulations using the model, are compared with experimental tests on PA66 and PP under different temperatures and different strain rates. Finally, a discussion and possible enhancements of the model are presented in section 4 .

In the text, bold symbols designate second or fourth-rank tensors, as indicated by the context. The contracted tensors products are expressed as:

$$
\boldsymbol{a}: \boldsymbol{b}=a_{i j} b_{j i},(\mathbb{A}: \boldsymbol{b})_{i j}=\mathbb{A}_{i j k l} b_{l k},
$$

where summation over a repeated index is supposed. The symbols $\mathbb{I}$ and $\boldsymbol{I}$ are 102 respectively the fourth and the second order symmetric identity tensors. The 
spherical and deviatoric fourth order operators $\mathbb{I}^{v o l}$ and $\mathbb{I}^{\text {dev }}$ are given by:

$$
\mathbb{I}^{v o l} \equiv \frac{1}{3} \boldsymbol{I} \otimes \boldsymbol{I} \text { and } \mathbb{I}^{d e v} \equiv \mathbb{I}-\mathbb{I}^{v o l},
$$

\section{Constitutive equations}

The model is formulated under the small perturbation hypothesis. The total 106 strain is decomposed into three parts: a viscoelastic (VE) strain $\varepsilon^{v e}$, a thermal (TH) strain $\varepsilon^{t h}$ and a viscoplastic (VP) one $\varepsilon^{v p}$ :

$$
\varepsilon=\varepsilon^{v e}+\varepsilon^{t h}+\varepsilon^{v p}
$$

108 The thermal strain is considered to be expressed as:

$$
\varepsilon^{t h}(T)=\underbrace{\left(T-T_{0}\right)}_{\theta} \alpha \boldsymbol{I}
$$

here $T$ is the absolute temperature at the current time and $T_{0}$ is the initial temperature, and $\alpha$ is the thermal expansion coefficient for an isotropic polymer, which may be temperature dependent for some polymers.

\subsection{Helmholtz free energy}

The proposed Helmholtz's free energy per unit mass $\psi$ is decomposed into a viscoelastic (VE) part denoted by strain energy function $\psi^{v e}$ and a hardening energy function $\psi^{h}$.

$$
\psi=\psi^{v e}+\psi^{h}
$$

${ }_{116}$ The VE part will be studied in sections 2.2 and 2.3. The hardening part of the energy is an extension of the expression defined in Doghri (1993) as:

$$
\rho \psi^{h}=\frac{1}{2} \chi(t, T): \chi(t, T)+\int_{0}^{p(t)} R(\xi, T) d \xi
$$


In this work, $\left(\varepsilon^{v p}, \boldsymbol{V}=\{p, \boldsymbol{\chi}\}\right)$ are internal variables, where the scalar variable $p$ models isotropic hardening and the strain-like tensor $\chi$ models kinematic hardening. Internal variables $\boldsymbol{V}$ are associated to thermodynamic forces $\boldsymbol{A}=\{R, \boldsymbol{X}\}$. The scalar variable $R$ measures the radius of the yield surface in the space of deviatoric stresses while the tensor variable $\boldsymbol{X}$ measures the translation of the center of that surface in the same space. The Cauchy stress 124 is denoted $\sigma$.

\subsection{Temperature-independent viscoelastic properties}

126

First we consider the case of temperature-independent viscoelastic properties. The expression of $\psi^{v e}$ is form-similar to the Helmholtz free energy proposed by Christensen and Naghdi (1967) for linear non-isothermal viscoelastic solids, but instead of the total strain in the purely VE latter formulation, we choose to write $\psi^{v e}$ in terms of the thermo-viscoelastic strain:

$$
\varepsilon^{t v e}=\varepsilon^{t h}+\varepsilon^{v e}=\varepsilon-\varepsilon^{v p}
$$

The expression of $\psi^{v e}$ is the following:

$$
\begin{aligned}
\rho \psi^{v e}=\rho \psi_{0}^{v e}+ & \int_{-\infty}^{t} \boldsymbol{D}(t-\tau): \frac{\partial \boldsymbol{\varepsilon}^{t v e}}{\partial \tau} d \tau-\int_{-\infty}^{t} \beta(t-\tau) \frac{\partial \theta}{\partial \tau} d \tau \\
& +\frac{1}{2} \int_{-\infty}^{t} \int_{-\infty}^{t}\left\{\frac{\partial \varepsilon^{t v e}(\tau)}{\partial \tau}: \mathbb{C}^{v e}(t-\tau, t-\eta): \frac{\partial \boldsymbol{\varepsilon}^{t v e}(\eta)}{\partial \eta}\right\} d \tau d \eta \\
& -\int_{-\infty}^{t} \int_{-\infty}^{t}\left\{\boldsymbol{\varphi}(t-\tau, t-\eta): \frac{\partial \varepsilon^{t v e}(\tau)}{\partial \tau} \frac{\partial \theta(\eta)}{\partial \eta}\right\} d \tau d \eta \\
& -\frac{1}{2} \int_{-\infty}^{t} \int_{-\infty}^{t}\left\{m(t-\tau, t-\eta) \frac{\partial \theta(\tau)}{\partial \tau} \frac{\partial \theta(\eta)}{\partial \eta}\right\} d \tau d \eta+O\left(\epsilon^{3}\right)
\end{aligned}
$$

where $\rho\left[\mathrm{kg} / \mathrm{m}^{3}\right]$ is the mass density, $\psi_{0}^{v e}$ is the Holmholtz free energy density of the material in the initial stress-free state. The functions $D, \beta, \mathbb{C}^{v e}, \varphi$ and $m$ are the relaxation mechanical functions, they are assumed to be continuous for arguments $\tau_{i}<0$; i.e.,

$$
D_{i j}\left(\tau_{1}\right)=0, \beta\left(\tau_{1}\right)=0, \mathbb{C}_{i j k l}^{v e}\left(\tau_{1}, \tau_{2}\right)=0, \varphi_{i j}\left(\tau_{1}, \tau_{2}\right)=0,
$$




$$
m\left(\tau_{1}, \tau_{2}\right)=0, \text { for } \tau_{1}<0 \text { and } \tau_{2}<0
$$

${ }_{138}$ The terms of $O\left(\epsilon^{3}\right)$ in equation 6 are neglected.

The Clausius-Duhem inequality requires the dissipation $\phi$ to be non-negative and reads as:

$$
\phi=\boldsymbol{\sigma}: \dot{\boldsymbol{\varepsilon}}-\rho(\dot{\psi}+S \dot{T})-\nabla T \cdot \frac{\boldsymbol{q}}{T} \geq 0
$$

where $\boldsymbol{q}$ is the heat flux vector and $S$ is the entropy per unit mass. After differentiation with respect to time of the free energy expression, using Leibnitz's rule, the Clausius-Duhem inequality leads to the following expressions of the stress and the entropy after canceling $D$ and $\beta$ terms (cf. AppendixA for details)

$$
\begin{aligned}
\boldsymbol{\sigma}(t) & =\int_{-\infty}^{t} \mathbb{C}^{v e}(t-\tau): \frac{\partial \varepsilon^{t v e}(\tau)}{\partial \tau} d \tau-\int_{-\infty}^{t} \boldsymbol{\varphi}(t-\tau) \frac{\partial \theta(\tau)}{\partial \tau} d \tau \\
\rho S(t) & =\int_{-\infty}^{t} m(t-\tau) \frac{\partial \theta(\tau)}{\partial \tau} d \tau+\int_{-\infty}^{t} \varphi(t-\tau): \frac{\partial \varepsilon^{t v e}(\tau)}{\partial \tau} d \tau
\end{aligned}
$$

The dissipation defined in equation 7 can be re-expressed as follows:

$$
\phi=\boldsymbol{\sigma}: \dot{\varepsilon}^{v p}-\rho \dot{\psi}^{h}+\Lambda-\nabla T \cdot \frac{\boldsymbol{q}}{T} \geq 0
$$
order term which can be neglected in front of the others. Consequently, and using the expression of $\psi^{h}$ (eq.4). The dissipation is rewritten as:

$$
\phi=-\nabla T \cdot \frac{\boldsymbol{q}}{T}+\underbrace{\boldsymbol{\sigma}: \dot{\boldsymbol{\varepsilon}}^{v p}-R \dot{p}-a \boldsymbol{\chi}(t): \dot{\chi}(t)}_{\phi_{\mathrm{mec}}} \geq 0
$$
where $\phi_{\text {mec }}$ is the mechanical dissipation transferred to heat. This effect is called self heating. Generally, it is important at high strain rates under monotonic loading or at high frequencies under cyclic loading. It can be the origin of important thermal softening that may lead to material failure.

154 The first law of thermodynamics can be expressed in the following form [See details in Doghri (2000), Chapter 12]: 


$$
\rho \dot{e}=\boldsymbol{\sigma}: \dot{\varepsilon}+\rho r_{e x t}-\operatorname{div} \boldsymbol{q}
$$

density of internal heat production due to external sources. Internal and free energies per unit mass, $e$ and $\psi$, are related by:

$$
\psi=e-T S
$$

Using the equations of state (and neglecting the $\Lambda$ term again) equation 12 can $\mathbf{1 6 0}$ be rewritten as follows:

$$
\rho T \dot{S}=\phi_{\mathrm{mec}}+\rho r_{e x t}-\operatorname{div} \boldsymbol{q}
$$

$\mathbf{1 6 2}$ This can again be rewritten as follows:

$$
\rho c_{p} \dot{T}=\left(\phi_{\mathrm{mec}}+\rho r_{e x t}-\rho T \mathcal{H}\right)-\operatorname{div} \boldsymbol{q}
$$

where $c_{p}[J / k g / K]$ is the specific heat capacity and $\mathcal{H}$ is the structural heating, given by the following expressions:

$$
\begin{aligned}
c_{p} & \equiv T \frac{\partial S}{\partial T} \\
\mathcal{H} & \equiv \frac{\partial S}{\partial \varepsilon^{t v e}}: \varepsilon^{t v e}
\end{aligned}
$$

According to Fourier's law, the heat conduction in an isotropic material can be expressed as:

$$
\boldsymbol{q}=-\boldsymbol{k} \nabla T
$$

The conductivity tensor $\boldsymbol{k}$ is assumed to be an isotropic tensor defined as $\boldsymbol{k}=k \boldsymbol{I}$.

\subsection{Temperature-dependent viscoelastic properties}

Let us start with purely VE case. When the properties are temperature170 dependent, then according to Schapery (1967) a thermodynamically valid constitutive relation is: 


$$
\boldsymbol{\sigma}(t)=\int_{-\infty}^{t} \mathbb{C}^{v e}(\bar{t}-\bar{\tau}): \frac{\partial \varepsilon(\tau)}{\partial \tau} d \tau-\int_{-\infty}^{t} \boldsymbol{\varphi}(\bar{t}-\bar{\tau}) \frac{\partial \theta(\tau)}{\partial \tau} d \tau
$$

172 where $\bar{t}$ and $\bar{\tau}$ are reduced times defined by

$$
\bar{t}=\int_{0}^{t} \frac{d \xi}{a_{T}(T(\xi))} ; \bar{\tau}=\int_{0}^{\tau} \frac{d \xi}{a_{T}(T(\xi))}
$$

with $a_{T}(T)$ being a temperature shift function. This formalism has been widely used since the 1960's. As noted also by Schapery 1967, eq 18 can be rewritten equivalently as:

$$
\boldsymbol{\sigma}(t)=\int_{-\infty}^{\bar{t}} \mathbb{C}^{v e}(\bar{t}-\bar{\tau}): \frac{\partial \varepsilon(\bar{\tau})}{\partial \bar{\tau}} d \bar{\tau}-\int_{-\infty}^{\bar{t}} \boldsymbol{\varphi}(\bar{t}-\bar{\tau}) \frac{\partial \theta(\bar{\tau})}{\partial \bar{\tau}} d \bar{\tau}
$$

This relation is form-identical to classical one for temperature-independent properties, provided that time is replaced by reduced time. Going back to coupled VE-VP, with temperature-dependent VE properties, we assume that the VE part of the free energy remains form-identical to that of equation (6) on the condition that the following substitutions are made:

$$
t \rightarrow \bar{t} ; \tau \rightarrow \bar{\tau} ; \eta \rightarrow \bar{\eta}
$$

The time derivative is given by

$$
\frac{\partial \bar{t}}{\partial t}=\frac{1}{a_{T}(T(t))}
$$

Using equations 21 and 22 , and following the same procedure as in section 2.2 and AppendixA, the following equations of state are found for the stress and the entropy:

$$
\begin{array}{r}
\boldsymbol{\sigma}(t)=\int_{-\infty}^{t} \mathbb{C}^{v e}(\bar{t}-\bar{\tau}): \frac{\partial \varepsilon^{t v e}(\tau)}{\partial \tau} d \tau-\int_{-\infty}^{t} \boldsymbol{\varphi}(\bar{t}-\bar{\tau}) \frac{\partial \theta(\tau)}{\partial \tau} d \tau \\
\rho S(t)=\int_{-\infty}^{t} m(\bar{t}-\bar{\tau}) \frac{\partial \theta(\tau)}{\partial \tau} d \tau+\int_{-\infty}^{t} \boldsymbol{\varphi}(\bar{t}-\bar{\tau}): \frac{\partial \varepsilon^{t v e}(\tau)}{\partial \tau} d \tau
\end{array}
$$


The dissipation equation (11) and the heat equation (15) remain unchanged ${ }_{186}$ (after neglecting the $\Lambda$ term). In the isotropic case, $\mathbb{C}^{v e}$ could be written as follows:

$$
\mathbb{C}^{\mathrm{ve}}(\bar{t})=2 G(\bar{t}) \mathbb{I}^{\text {dev }}+3 K(\bar{t}) \mathbb{I}^{v o l}
$$

188

where $G(\bar{t})$ and $K(\bar{t})$ are shear and bulk relaxation functions, respectively, which can be expressed using the Prony series:

$$
G(\bar{t})=G_{\infty}+\sum_{i=1}^{I} G_{i} \exp \left(-\frac{\bar{t}}{g_{i}}\right) ; K(\bar{t})=K_{\infty}+\sum_{j=1}^{J} K_{j} \exp \left(-\frac{\bar{t}}{k_{j}}\right)
$$

Here, $g_{i}(i=1 . . I)$ and $k_{j}(j=1 . . J)$ are the deviatoric and volumetric relaxation times respectively; $G_{i}(i=1 . . I)$ and $K_{j}(j=1 . . J)$ are the corresponding moduli or weights, and $G_{\infty}$ and $K_{\infty}$ are the long-term elastic shear and bulk moduli.

We recall that for the isotropic case, the thermal expansion is defined by equation 2. After replacing the thermo-viscoelastic strain by its expression and in order to obtain familiar expression for the stress, an assumption was made for the function $\varphi$ to be defined as $\varphi_{i j}(t)=\varphi(t) \delta_{i j}=3 \alpha K(\bar{t}) \delta_{i j}$. The following expressions for stress and entropy are obtained (cf. AppendixA for details). The Cauchy stress is divided into deviatoric and hydrostatic parts:

$$
\boldsymbol{\sigma}(t)=\boldsymbol{s}(t)+\sigma_{H}(t) \mathbf{1}
$$

where

$$
\left\{\begin{array}{l}
s_{i j}(t)=2 \int_{-\infty}^{t} G(\bar{t}-\bar{\tau}) \frac{\partial \boldsymbol{\xi}_{i j}^{v e}(\tau)}{\partial \tau} d \tau \\
\sigma_{H}(t)=3 \int_{-\infty}^{t} K(\bar{t}-\bar{\tau}) \frac{\partial \epsilon_{H}^{v e}(\tau)}{\partial \tau} d \tau
\end{array}\right.
$$

200

with the viscoelastic strain tensor is also divided into deviatoric and dilatational parts:

$$
\varepsilon^{v e}(t)=\boldsymbol{\xi}^{v e}(t)+\epsilon_{H}^{v e}(t) \mathbf{1}
$$

The equation 28 is equivalent to: 


$$
\boldsymbol{\sigma}(t)=\int_{-\infty}^{t} \mathbb{C}^{v e}(\bar{t}-\bar{\tau}): \frac{\partial \varepsilon^{v e}(\tau)}{\partial \tau} d \tau
$$

The entropy is then expressed as

$$
\rho S(t)=\int_{-\infty}^{t}\left(m(\bar{t}-\bar{\tau})+9 \alpha^{2} K(\bar{t}-\bar{\tau})\right) \frac{\partial \theta(\tau)}{\partial \tau} d \tau
$$

\subsection{Viscoplastic flow rules}

Using the generalized normality as in (Krairi and Doghri, 2014; Chaboche, 206 1997), we have the following evolution laws:

$$
\dot{\boldsymbol{\varepsilon}}^{v p}=\dot{\gamma} \frac{\partial F}{\partial \boldsymbol{\sigma}}, \dot{\boldsymbol{V}}=\dot{\gamma} \frac{\partial F}{\partial \boldsymbol{A}},
$$

here $\dot{\gamma}$ is a viscoplastic multiplier and the potential $F$ is the non-isothermal 208 extension of the one proposed by (Lemaitre and Chaboche, 1994):

$$
F(\boldsymbol{\sigma}, R, \boldsymbol{X}, T)=f(\boldsymbol{\sigma}, R, \boldsymbol{X}, T)+\frac{b}{2 a} \boldsymbol{X}: \boldsymbol{X}
$$

where $f(\boldsymbol{\sigma}, R, \boldsymbol{X}, T)$ represents the viscoelastic domain if $f<0$, and viscoplas210 tic flow if $f>0$. The following expression for $f$ is considered:

$$
f(\boldsymbol{\sigma}, R, \boldsymbol{X}, T)=(\boldsymbol{\sigma}-\boldsymbol{X})_{e q}-\sigma_{Y}(T)-R(T, p)
$$

here $\sigma_{Y}(T)$ is the viscoelastic limit at a given temperature $(T)$ and $(\boldsymbol{\sigma}-\boldsymbol{X})_{e q}$ ${ }_{212}$ is chosen as the von Mises measure of $(\boldsymbol{\sigma}-\boldsymbol{X})$ :

$$
(\boldsymbol{\sigma}-\boldsymbol{X})_{e q}=\left[\frac{3}{2}(\boldsymbol{s}-\boldsymbol{X}):(\boldsymbol{s}-\boldsymbol{X})\right]^{1 / 2}
$$

here $s$ is the deviatoric part of $\boldsymbol{\sigma}$. Using (eq. 32), the following evolution 214 equations are found :

$$
\begin{aligned}
\dot{\boldsymbol{\varepsilon}}^{v p} & =\dot{\gamma} \boldsymbol{N}, \\
\dot{p} & =\dot{\gamma}, \\
\dot{\chi} & =\dot{\gamma}\left(\boldsymbol{N}-\frac{b}{a} \boldsymbol{X}\right)
\end{aligned}
$$


where the following notation was introduced :

$$
\boldsymbol{N} \equiv \frac{\partial f}{\partial \boldsymbol{\sigma}}=\frac{3}{2} \frac{s}{\sigma_{e q}}
$$

${ }_{216}$ Since $\dot{\boldsymbol{\varepsilon}}^{v p}$ and $\boldsymbol{N}$ are deviatoric and $(\boldsymbol{N}: \boldsymbol{N}=3 / 2)$, the accumulated viscoplastic strain rate $\dot{p}$ is related to $\dot{\varepsilon}^{v p}$ by :

$$
\dot{p}=\left(\frac{2}{3} \dot{\varepsilon}^{v p}: \dot{\varepsilon}^{v p}\right)^{1 / 2}
$$

218

and it is defined by:

$$
\begin{cases}\text { if } f \leqslant 0 & \dot{p}=0 \\ \text { if } f>0 & \dot{p}=g_{v}\left(\sigma_{e q}, p, T\right)>0\end{cases}
$$

where $g_{v}$ is the viscoplastic function.

\subsection{Summary of constitutive equations}

In summary, the main constitutive equations are the following:

$$
\left\{\begin{aligned}
\varepsilon & =\varepsilon^{t h}+\boldsymbol{\varepsilon}^{v e}+\boldsymbol{\varepsilon}^{v p} \\
\varepsilon^{v e} & =\boldsymbol{\xi}^{v e}+\epsilon_{H}^{v e} \mathbf{1} \\
s_{i j}(t)= & 2 \int_{-\infty}^{t} G(\bar{t}-\bar{\tau}) \frac{\partial \boldsymbol{\xi}_{i j}^{v e}(\tau)}{\partial \tau} d \tau \\
\sigma_{H}(t)= & 3 \int_{-\infty}^{t} K(\bar{t}-\bar{\tau}) \frac{\partial \epsilon_{H}^{v e}(\tau)}{\partial \tau} d \tau \\
= & \frac{3}{2} \frac{(\boldsymbol{s}-\boldsymbol{X})}{(\boldsymbol{\sigma}-\boldsymbol{X})_{e q}} \dot{p}, \\
& f(\boldsymbol{\sigma}, R, \boldsymbol{X}, T)=(\boldsymbol{\sigma}-\boldsymbol{X})_{e q}-\sigma_{Y}(T)-R(T, p) \\
& \text { if } f>0 \dot{p}=g_{v}\left(\sigma_{e q}, p, T\right)>0 \text { otherwise } \dot{p}=0 \\
& \dot{\boldsymbol{X}}=\left(a \dot{\boldsymbol{\varepsilon}}^{v p}-b \boldsymbol{X} \dot{p}\right) \\
\rho S(t)= & \int_{-\infty}^{t}\left(m(\bar{t}-\bar{\tau})+9 \alpha^{2} K(\bar{t}-\bar{\tau})\right) \frac{\partial \theta(\tau)}{\partial \tau} d \tau
\end{aligned}\right.
$$

222 The dissipation is expressed by equation 11 and the heat equation is given by equation 15. It should be noted that using the von Mises yield criterion makes 
the viscoplastic behavior insensitive to change in hydrostatic pressure. However the thermo-viscoelastic response should be influenced. This assumption is further discussed in section 4 .

In the above equations the concept of reduced time is employed as in Schapery (1969), however we are using a generalized shift function $A_{S h}$ which allows to take into account the aging and the moisture effect, such that the reduced time $(\bar{t})$ is related to the real time $(t)$ by the following expression:

$$
\bar{t}=\int_{0}^{t} \frac{d t^{\prime}}{A_{S h}\left(t^{\prime}\right)}
$$

with $A_{S h}$ defined as follows:

$$
A_{S h}=a_{T} a_{m} a_{a}
$$

where $a_{T}$ is representing the effect of temperature, $a_{m}$ is representing the effect of moisture and $a_{a}$ is representing the time shift due to material aging.

\section{Experimental validation}

In order to validate the proposed thermo-mechanical model against experimental tests, it was implemented into the commercial finite element code ABAQUS by combining user subroutines UMAT and UMATHT. The subroutine UMAT allows to define the stress-strain response and the heat production from mechanical dissipation at each integration point, based on an implicit numerical algorithm following the methods in (Simo and Hughes, 1998; Doghri, 2000). At each time increment, the subroutine UMAT compute heat generated due to dissipation through variable RPL. The temperature field is computed by ABAQUS as part of an iterative solution where UMATHT provides the heat flux vector and the thermal constitutive behavior. In the following, the thermo-mechanical behavior of Polyamide 66 and Polyproylene were studied. FE models for the specimens used to perform the experimental characterization were created and the numerical simulation results are compared to experimental data. Two type

244 of specimens are modeled and simulated by finite elements: tensile samples and shear samples. For the tensile samples which have rectangular and circular cross 
246

258

sections, based on the symmetry of the geometry and loading symmetry only fourth of the sample is modeled and meshed. Symmetry boundary conditions are applied on the 3 symmetry surfaces. A controlled displacement is applied on the clamps specimen shoulders contact surface.However, for the shear sample the whole geometry sample according to the standard ASTM D 5379. The initial testing temperature is defined as a predefined field throughout the model.

252 The convection heat transfer is not taken into account since the external environmental conditions (i.e ambient air temperature and convection coefficient) are not known. The FE models are meshed using element C3D20T, a 20 node triquadratic displacement and trilinear temperature brick element.

256 The selected models and functions for the numerical simulations are the following: The kinematic hardening is assumed to be negligible in the studied cases. For the shift function $\left(A_{s h}\right), a_{m}$ and $a_{a}$ are equal to 1 , since constant relative humidity and no effect of aging are assumed. The function $a_{T}$ is the Wo Williams-Landel-Ferry (WLF) equation defined as:

$$
\log \left(a_{T}\right)=-\frac{C_{1}\left(T-T_{r e f}\right)}{C_{2}+\left(T-T_{r e f}\right)}
$$

For the viscoplastic behavior,

$$
\left\{\begin{array}{l}
\sigma_{y}(T)=\Gamma(T) \sigma_{y, r e f} \\
R(T, p)=\Gamma(T) R\left(T_{r e f}, p\right)
\end{array}\right.
$$

with $\sigma_{y, r e f}$ is a yield stress at a reference temperature $T_{r e f}$, it represents the limit of linear viscoelastic response of the material in term of stress. $R\left(T_{r e f}, p\right)$ corresponds to the isotropic hardening with material parameters identified at the reference temperature. $\Gamma(T)$ is a temperature sensitivity function expressed as:

$$
\Gamma(T)=\Gamma(\beta, T)=\exp \left(-\beta\left(T-T_{r e f}\right)\right)
$$

$\beta$ is a material parameter, $\Gamma\left(T_{\text {ref }}\right)=1$, this function is inspired from the observation made by Zhou and Mallick (2002) on polypropylene (PP) and talcfilled polypropylene under different strain rates and different temperatures. The 
power law is selected for the viscoplastic function:

$$
g_{v}=\frac{\sigma_{y}}{\eta}\left(\frac{f}{\sigma_{y}}\right)^{m}
$$

$\eta$ is a variable that is taken constant for PP, since the temperature sensitivity of $\sigma_{y}$ is enough to capture the temperature sensitivity of the viscoplastic behavior. For PA66, $\eta$ is considered to be temperature dependent.

For each material PA66 and PP, the needed material parameters are the viscoelastic parameters (Table 1 for PA66 and Table 3 for PP), the viscoplastic parameters (Table 2 for PA66 and Table 4 for PP) and the WLF parameters, in addition to thermal properties which are given in the text in each corresponding section.

\subsection{Case of Polyamide 66 (PA66)}

Baquet (2011) and Maurel-Pantel et al. (2015) reported the results of thermomechanical uniaxial tensile and shear experimental tests on PA66 under different strain rates and temperatures. Digital image correlation (DIC) technique was used by the authors in order to analyze the deformation field on the front surface of the samples. The authors also presented measurements of the self heating at the specimen's external surface. They employed an infrared camera (see Maurel-Pantel et al. (2011) for more details about the employed procedure). The material was supplied by Solvay Performance Polyamides, it was conditioned until equilibrium was reached with an air containing $50 \%$ of relative humidity during the experiment, because of the high influence of relative humidity on the Polyamide material.

The proposed model is employed to simulate the material thermo-mechanical response and its self heating. In order to identify the viscoelastic properties, DTMA is employed. It was performed at temperatures between $-100^{\circ} \mathrm{C}$ and $210^{\circ} \mathrm{C}$. For the current study, the selected range was $0^{\circ} \mathrm{C}$ to $100^{\circ} \mathrm{C}$. Master curves for the storage and loss moduli were built in order to take into account the effect of time and temperature simultaneously (cf. figure 1). These curves were used in order to identify the Prony series coefficients. Several numbers of terms in the Prony series were tested and a minimum number of 20 terms was found to be needed in order to fit the master curve correctly. The instantaneous modulus $E_{0}$ is not given for confidentiality reasons, the Poisson's ratio 
is assumed to be constant and equal to $\nu=0.42$ and the identified viscoelastic parameters are listed in table 1. It should be noted that the Poisson's ratio is generally not constant for thermoplastic polymers and that the use of shear and bulk time functions, expressed using Prony series determined directly from experiments is more appropriate to model the multiaxial aspects of material behavior. However, if the only available experimental data are uniaxial tests, then an estimate of the shear and bulk moduli may be found, assuming a constant Poisson's ratio. With the latter assumption, parameters $G_{i}, K_{i}, g_{i}$ and $k_{j}$ are obtained by the following relations :

$$
\begin{cases}G_{\infty}=\frac{E_{\infty}}{2(1+\nu)} ; G_{i}=\frac{E_{i}}{2(1+\nu)} \quad ; g_{i}=\frac{\tau_{i} E_{i}}{G_{i}} \\ K_{\infty}=\frac{E_{\infty}}{3(1-2 \nu)} ; K_{i}=\frac{E_{i}}{3(1-2 \nu)} \quad ; k_{i}=\frac{\tau_{i} E_{i}}{K_{i}}\end{cases}
$$

More details about the viscoelastic material parameter identification can be found in Krairi and Doghri (2014). The parameters for the WLF equation are identified using the shift factors at each temperature employed to construct the master curves (cf. figure 2), $T_{r e f}=25^{\circ} \mathrm{C}, C_{1}=26.21$ and $C_{2}=153.16^{\circ} \mathrm{C}$. The material density, the thermal expansion coefficient, the thermal conductivity and the specific heat capacity are $\rho=1140 \mathrm{~kg} \mathrm{~m}^{-3}, \alpha=70.10^{-6} \mathrm{~K}^{-1}, k=$ 316 $0.27 \mathrm{~W} /(\mathrm{mK})$ and $c=1670 \mathrm{~J} \mathrm{~K}^{-1} \mathrm{~kg}^{-1}$, respectively.

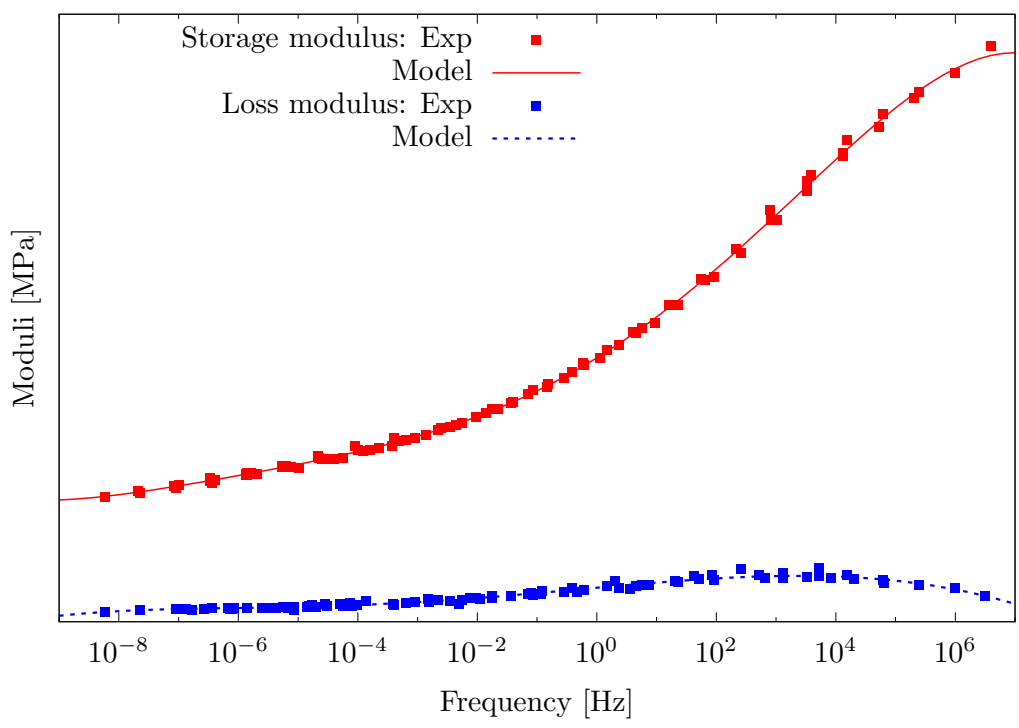

Figure 1: Master curve for PA66 conditioned at 50\% 


\begin{tabular}{|c|c|c|c|c|c|c|c|c|c|c|}
\hline$i$ & 1 & 2 & 3 & 4 & 5 & 6 & 7 & 8 & 9 & 10 \\
\hline $\log \left(\tau_{i}\right)$ & -6.59 & -5.80 & -5.01 & -4.22 & -3.42 & -2.63 & -1.84 & -1.05 & -0.26 & 0.53 \\
\hline$E_{i} / E_{0}$ & 0.07 & 0.073 & 0.076 & 0.078 & 0.077 & 0.073 & 0.068 & 0.062 & 0.056 & 0.050 \\
\hline \hline$i$ & 11 & 12 & 13 & 14 & 15 & 16 & 17 & 18 & 19 & 20 \\
\hline $\log \left(\tau_{i}\right)$ & 1.32 & 2.12 & 2.91 & 3.70 & 4.49 & 5.28 & 6.07 & 6.87 & 7.66 & 8.45 \\
\hline$E_{i} / E_{0}$ & 0.027 & 0.023 & 0.019 & 0.016 & 0.014 & 0.014 & 0.015 & 0.018 & 0.024 & 0.034 \\
\hline
\end{tabular}

Table 1: Identified VE parameters for PA66 at $\mathrm{T}=23^{\circ} \mathrm{C}$

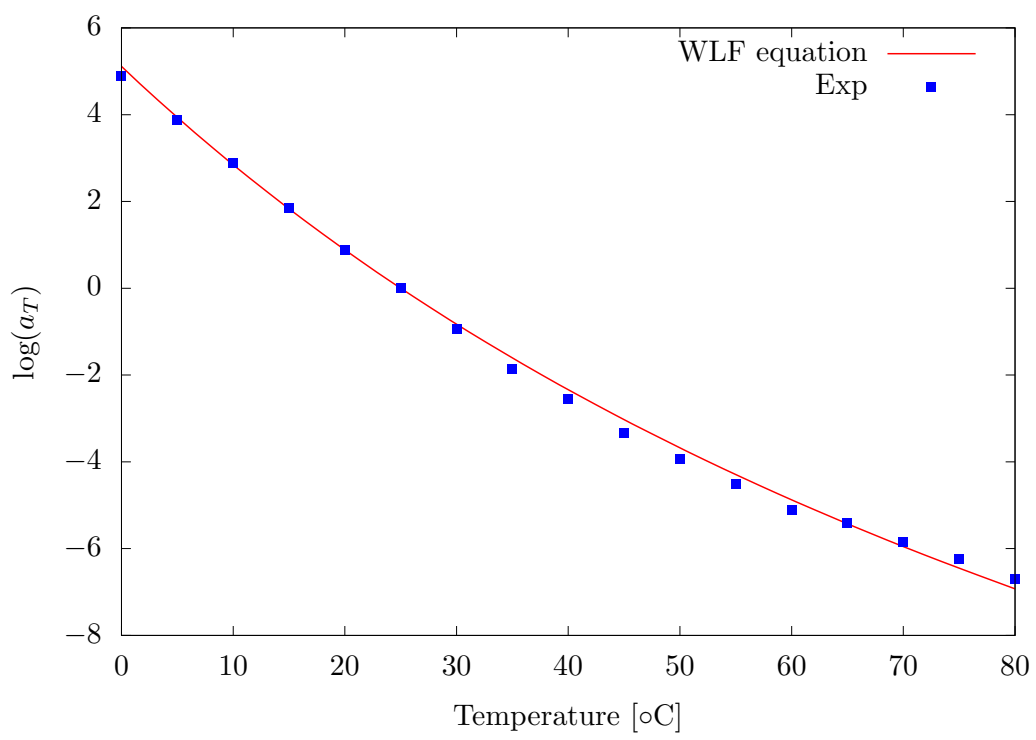

Figure 2: Shift factors for PA66 conditioned at 50\%, employed to calibrate the WLF shift function

The experimental stress strain curves at different strain rates at temperatures $T=21.5^{\circ} \mathrm{C}$ and $T=26{ }^{\circ} \mathrm{C}$ (See figures 6 and 3 ) are used to identify the parameters for the yield stress, the isotropic hardening and the viscoplastic function, and to calibrate the function $\Gamma(T)$. The identified viscoplastic material parameters are listed in table 2. In the following, the stress-strain curves for PA66 are plotted in terms of normalized stress (true stress divided by a constant stress $\left.324 \quad \sigma_{0}\right)$ for confidentiality reasons. 
The predicted stress strain curves at temperatures $T=40^{\circ} \mathrm{C}$ and $T=60^{\circ} \mathrm{C}$ are compared to the experimental ones in figures 7 and 8 . An acceptable agreement can be seen between the experimental data and numerical predictions.

${ }_{328}$ The average self-heating at the specimens surface is predicted at temperatures $T=21.5^{\circ} \mathrm{C}$ and $T=26^{\circ} \mathrm{C}$. From figure 3, we can see that the self heating is well predicted at $T=26^{\circ} \mathrm{C}$ and a strain rate $\dot{\varepsilon}=2.410^{-1} \mathrm{~s}^{-1}$, the field of temperature is plotted in figure 4 at a strain of $25 \%$. For lower strain rate $\dot{\varepsilon}=2.410^{-3} \mathrm{~s}^{-1}$ at the same temperature a slight over-estimation of predicted rise of temperature can be seen in figure 5 , but it is still within the measurement noise. It seems that at small strain rates, the increase of the temperature is small, therefore it is difficult to obtain accurate experimental measurements. In figure 6 , for the case of the temperature $T=21.5^{\circ} \mathrm{C}$ and strain rate $\dot{\varepsilon}=1.810^{-2} \mathrm{~s}^{-1}$, a drop of temperature occurred mainly when the material response is linear, but when the nonlinear regime is dominant, an increase of the temperature can be seen. The model is able to predict the increase and temperature with a small overestimation for this case.

$$
\begin{aligned}
& \text { Yield stress: } \sigma_{y}(T)=\Gamma\left(\beta_{1}, T\right) \sigma_{y, r e f} \\
& \quad T_{r e f}=25^{\circ} \mathrm{C}, \beta_{1}=0.011, \sigma_{y, r e f}=15.5 \mathrm{MPa} \\
& \text { Isotropic hardening: } R(T, p)=\Gamma\left(\beta_{1}, T\right) k p^{n} \\
& \quad k=103 \mathrm{MPa}, n=0.32 \\
& \text { Viscoplastic function: } g_{v}=\frac{\sigma_{y}}{\eta}\left(\frac{f}{\sigma_{y}}\right)^{m} \text { with } \eta=\eta_{0} \Gamma\left(\beta_{2}, T\right) \\
& \quad \eta_{0}=74 \mathrm{MPa} . \mathrm{s}, m=2, \beta_{2}=0.07
\end{aligned}
$$

Table 2: Viscoplastic parameters for PA66 


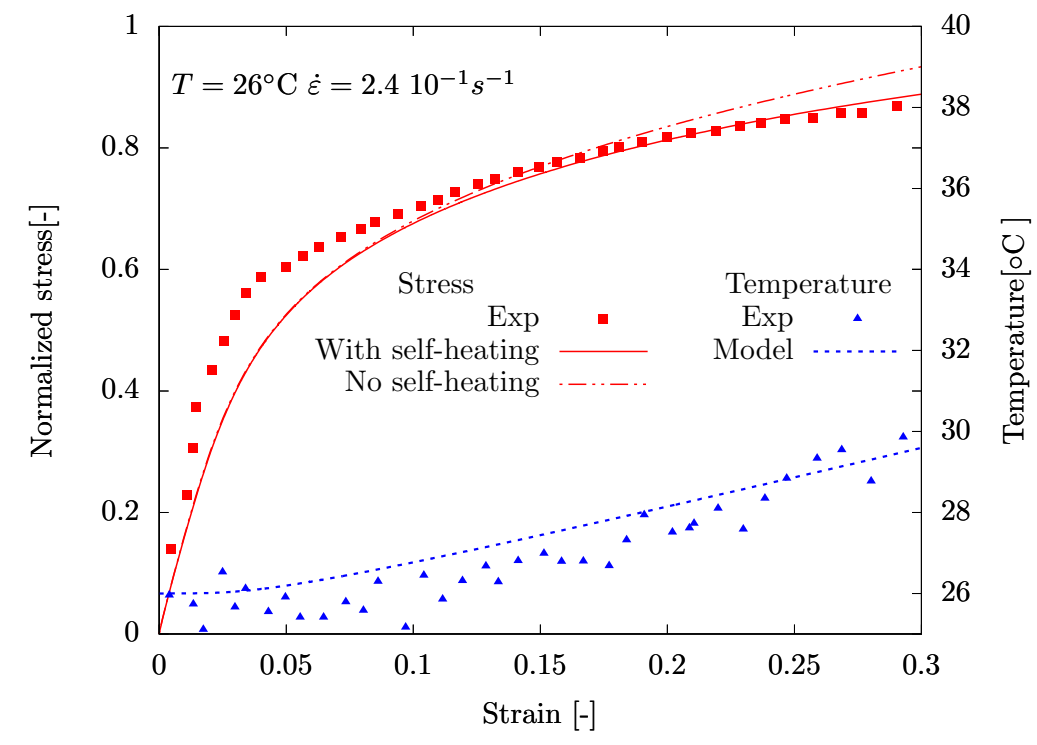

Figure 3: Tensile tests under a strain rate $\dot{\varepsilon}=2.410^{-1} \mathrm{~s}^{-1}$ at the temperature $T=26^{\circ} \mathrm{C}$
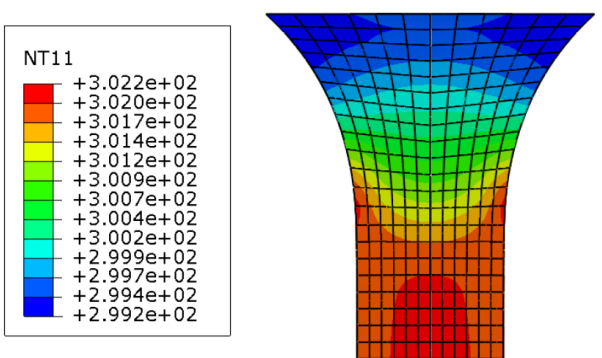

Figure 4: An example of the self-heating field within the dogbone specimen of PA66 under uniaxial test at $T=26^{\circ} \mathrm{C}$ and a strain rate $\dot{\varepsilon}=2.410^{-1} \mathrm{~s}^{-1}$. The average strain is equal to 0.25 . The values of temperature are in ${ }^{\circ} \mathrm{K}$. 


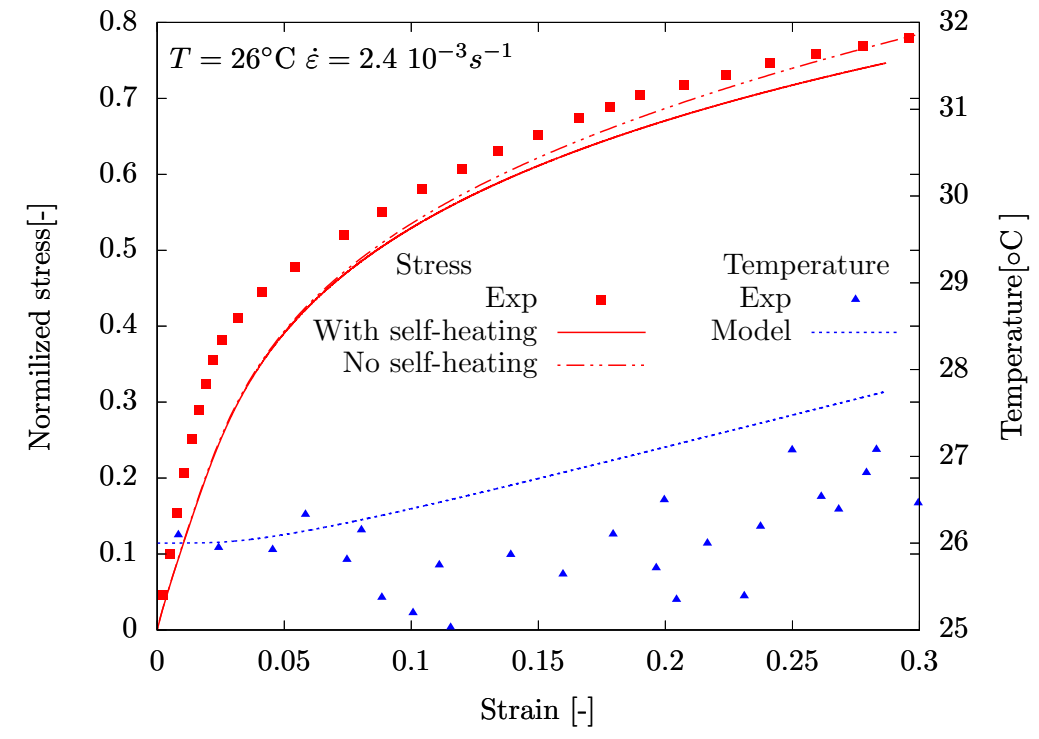

Figure 5: Tensile test under a strain rate $\dot{\varepsilon}=2.410^{-3} \mathrm{~s}^{-1}$ at the temperature $T=26^{\circ} \mathrm{C}$

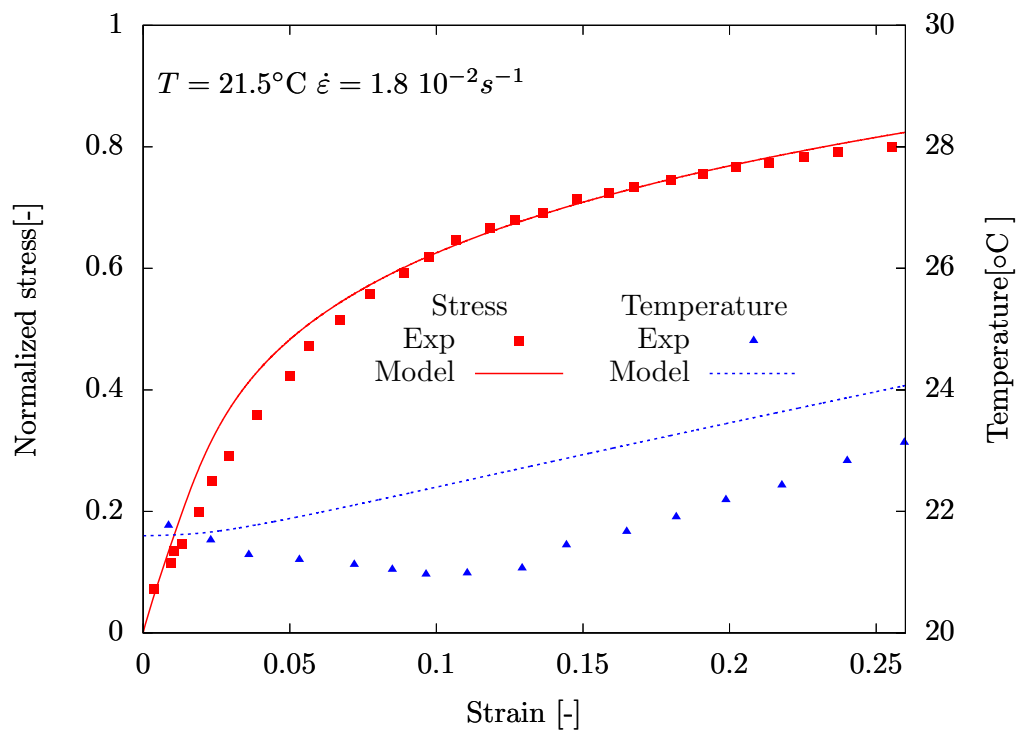

Figure 6: Tensile test under a strain rate $\dot{\varepsilon}=1.810^{-2} \mathrm{~s}^{-1}$ at the temperature $T=21.5^{\circ} \mathrm{C}$ 


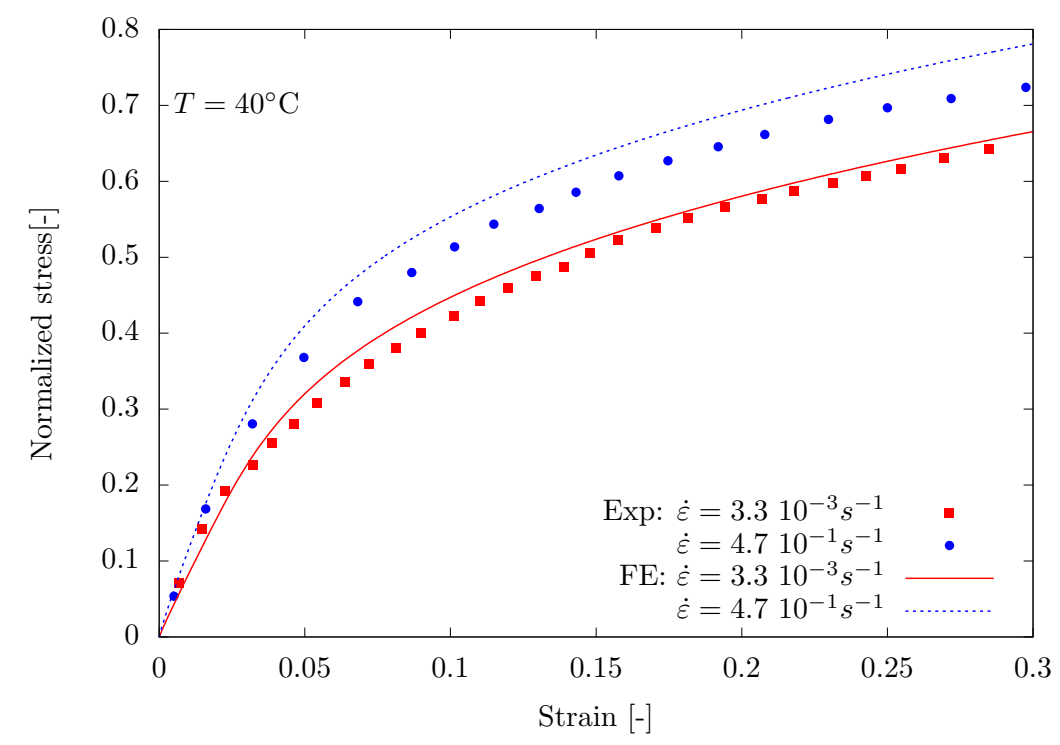

Figure 7: Tensile tests under different strain rates at the temperature $T=40^{\circ} \mathrm{C}$

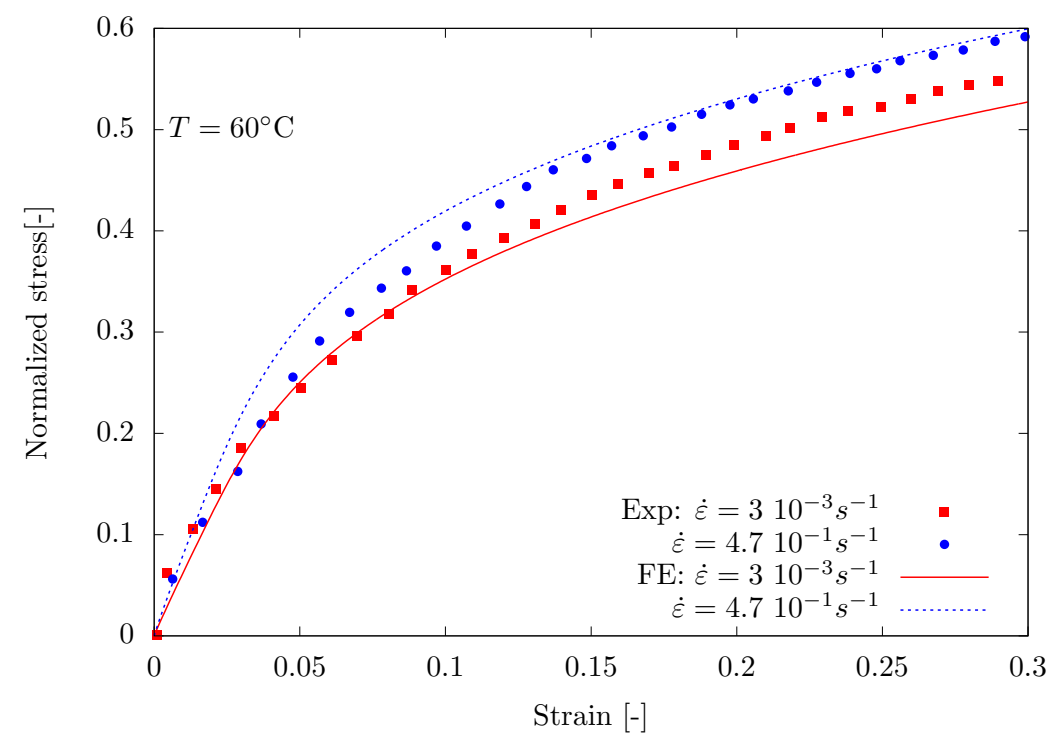

Figure 8: Tensile tests under different strain rates at the temperature $T=60^{\circ} \mathrm{C}$

342 The proposed model is also employed to predict the behavior of PA66 under shear loading using Iosipescu configuration (described in standard "ASTM 344 D 5379"), with different strain rates at temperature $T=29^{\circ} \mathrm{C}$. The model predictions are compared to experimental data and numerical simulations of 
346 Maurel-Pantel et al. (2015) in figures 9, 10, 11 and 12. It is seen that the proposed model better captures the material response under shear loading as 348 compared to Maurel-Pantel et al. (2015) model.

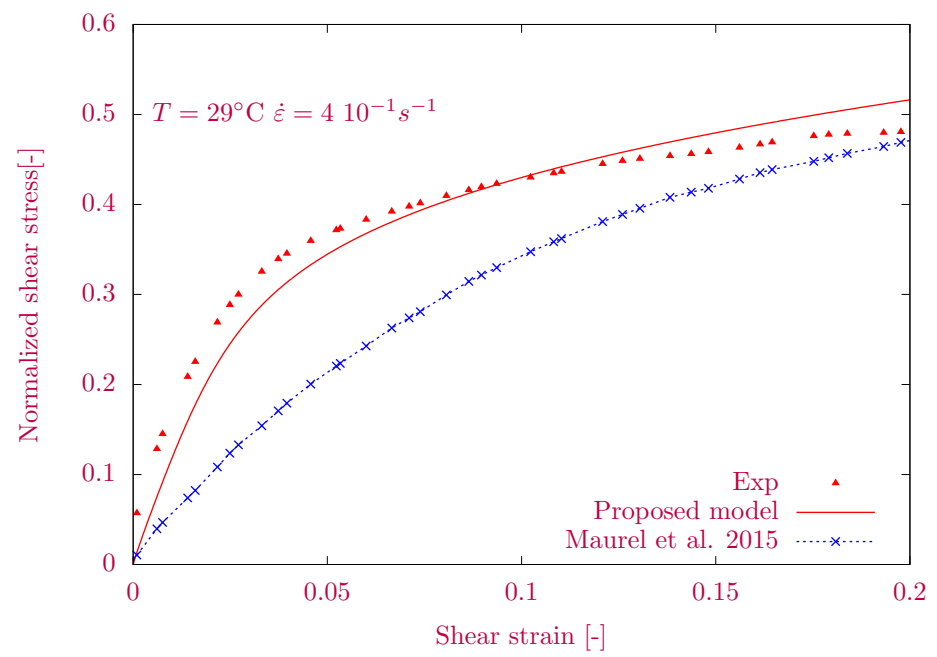

Figure 9: Shear test under a strain rate $\dot{\varepsilon}=4.010^{-1} \mathrm{~s}^{-1}$ at temperature $T=29^{\circ} \mathrm{C}$

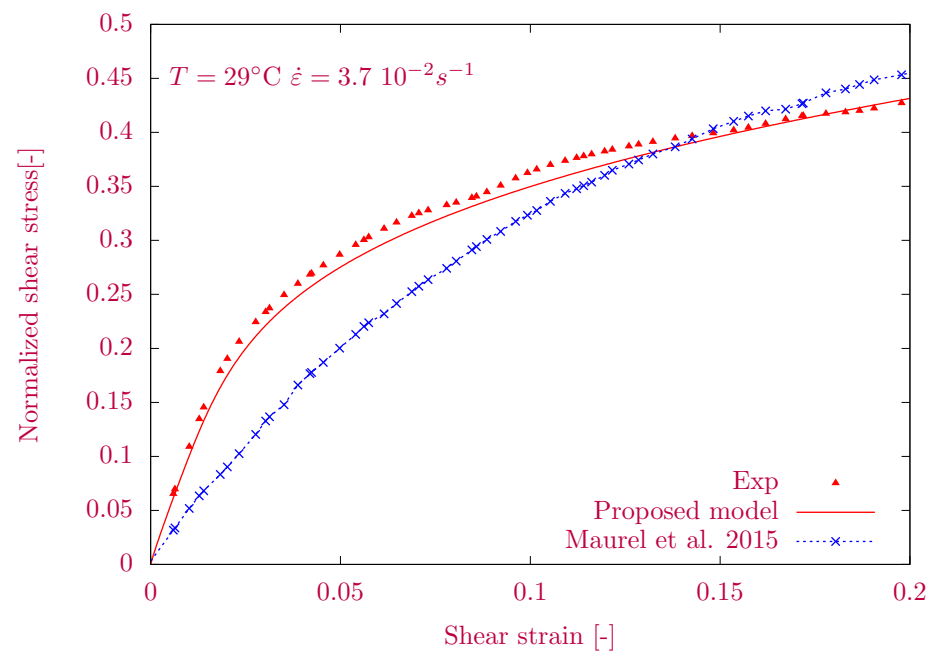

Figure 10: Shear test under a strain rate $\dot{\varepsilon}=3.710^{-2} \mathrm{~s}^{-1}$ at temperature $T=29^{\circ} \mathrm{C}$ 


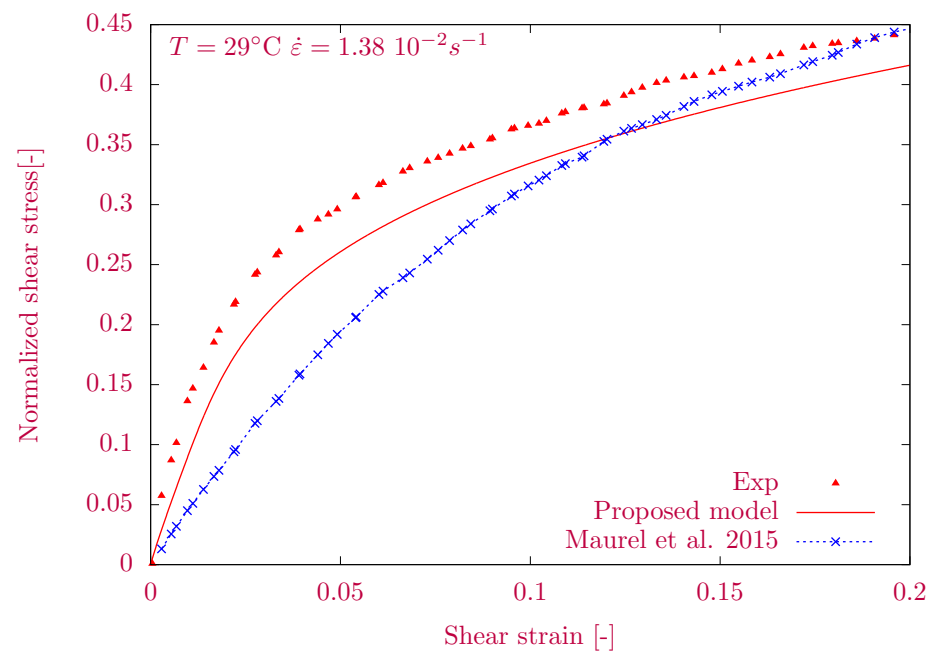

Figure 11: Shear tests under a strain rate $\dot{\varepsilon}=1.3810^{-2} \mathrm{~s}^{-1}$ at temperature $T=29^{\circ} \mathrm{C}$ 352

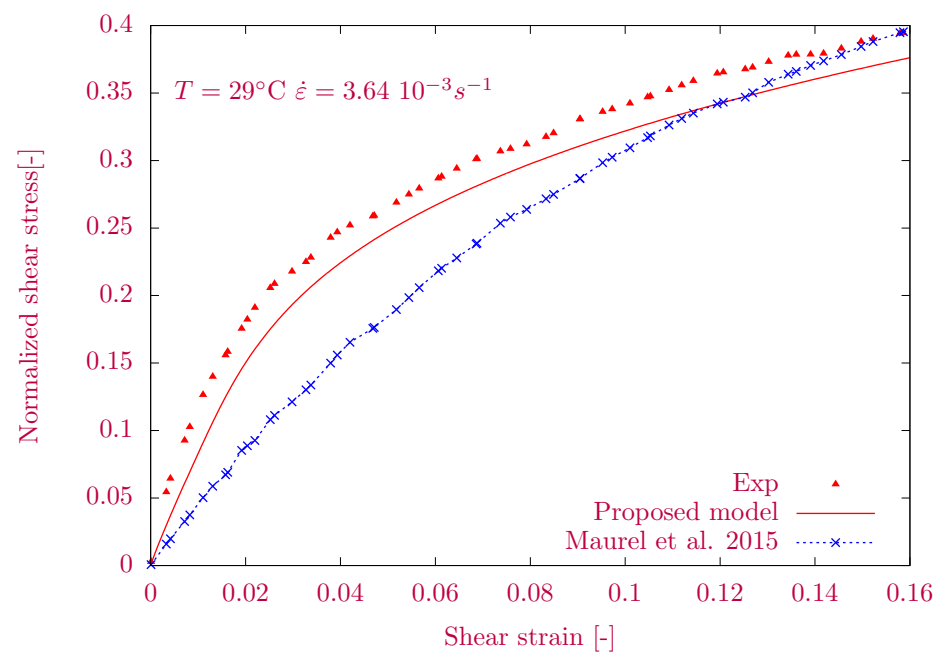

Figure 12: Shear tests under a strain rate $\dot{\varepsilon}=3.6410^{-3} \mathrm{~s}^{-1}$ at temperature $T=29^{\circ} \mathrm{C}$ 


\subsection{Case of polypropylene (PP)}

354

356

358

370

In this section, experimental uniaxial tests were performed on injected polypropylene (PP 575P from Sabic) at different temperatures and strain rates to calibrate and validate the proposed constitutive material model. The digital image correlation (DIC) technique was employed in order to measure the local field of deformation during the loading. The strain measurement was verified with an extensometer at room temperature. The temperature was controlled by a thermocouple inside the heat chamber. A second measurement was performed with a K-type thermocouple directly next to the sample. Dogbone-shaped samples according to DIN EN ISO 527-2, type 1A were tested on an Instron 5800 tensile machine. The true strain was calculated with the correlation software VIC3D, using the logarithmic Hencky tensor.

Similar to the case of PA66, DMA was used in order to identify the viscoelastic properties (i.e. Prony series coefficients). Injected PP was grinded and polished with water cooling to a thickness of $3 \mathrm{~mm}$ and dried afterwards in a desiccator until constant weight. According to DSC results, the crystallinity remained unchanged. On a TA Q800 frequency sweeps of $0.1 \%$ strain were performed using a dual cantilever clamp at frequencies between $1 \mathrm{~Hz}$ and 50 $\mathrm{Hz}$. In steps of $5^{\circ} \mathrm{C}$ with an isothermal step length of $15 \mathrm{~min}$ to ensure uniform temperature distribution, the temperature was increased from $23^{\circ} \mathrm{C}$ to $60^{\circ} \mathrm{C}$. From the results, master curves for loss and storage moduli were constructed (cf. figure 13). The instantaneous modulus is $E_{0}=2000 \mathrm{MPa}$, the Poisson's ratio is assumed to be constant and equal to $\nu=0.42$ and the identified viscoelastic parameters are listed in Table 3. The parameters for the WLF equation are $T_{r e f}=23^{\circ} \mathrm{C}, C_{1}=61.22$ and $C_{2}=178.5^{\circ} \mathrm{C}$, identified using the 38 experimental shift factors (cf figure 14). The material density, the thermal expansion coefficient, the thermal conductivity and the specific heat capacity are $\rho=943 \mathrm{~kg} \mathrm{~m}^{-3}, \alpha=90.10^{-6} \mathrm{~K}^{-1}, \mathrm{k}=0.2 \mathrm{~W} /(\mathrm{mK})$ and $c_{p}=1920 \mathrm{~J} \mathrm{~K}^{-1} \mathrm{~kg}^{-1}$, respectively. 


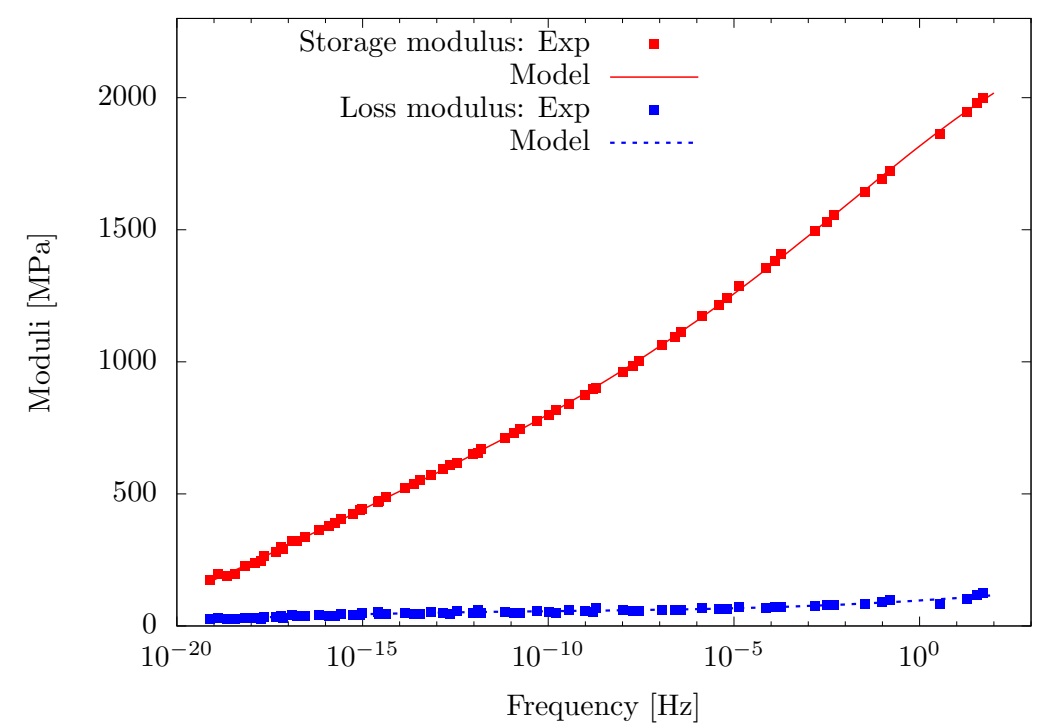

Figure 13: Master curves for PP

\begin{tabular}{|c|c|c|c|c|c|c|c|c|c|c|}
\hline$i$ & 1 & 2 & 3 & 4 & 5 & 6 & 7 & 8 & 9 & 10 \\
\hline $\log \left(\tau_{i}\right)$ & -1.70 & -0.55 & 0.59 & 1.73 & 2.88 & 4.02 & 5.17 & 6.31 & 7.46 & 8.60 \\
\hline$E_{i}[\mathrm{MPa}]$ & 163 & 122 & 126 & 129 & 126 & 119 & 112 & 105 & 99 & 93.5 \\
\hline \hline$i$ & 11 & 12 & 13 & 14 & 15 & 16 & 17 & 18 & 19 & 20 \\
\hline $\log \left(\tau_{i}\right)$ & 9.74 & 10.9 & 12.0 & 13.1 & 14.3 & 15.5 & 16.6 & 17.8 & 18.9 & 20.0 \\
\hline$E_{i}[\mathrm{MPa}]$ & 88.8 & 84.6 & 81.4 & 79.7 & 79.8 & 80.4 & 77.4 & 64.1 & 51.1 & 91.7 \\
\hline
\end{tabular}

Table 3: Identified viscoelastic parameters for $\mathrm{PP}$ at $\mathrm{T}=23^{\circ} \mathrm{C}$ 


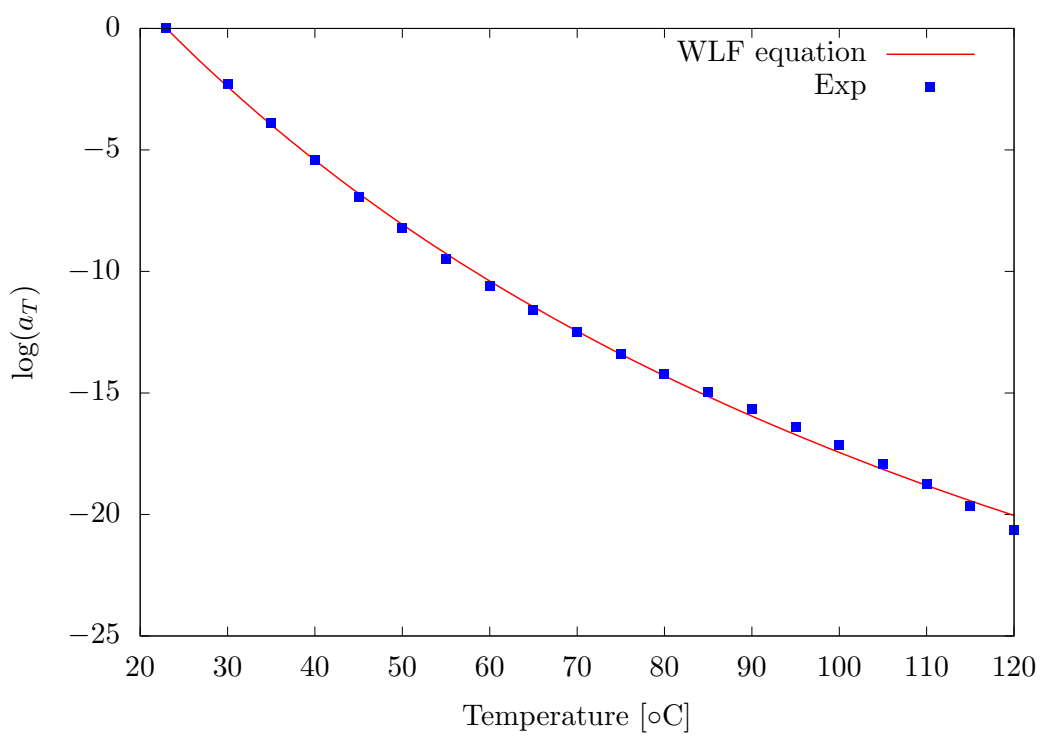

Figure 14: Shift factors for PP, employed to calibrated the WLF shift function

The experimental stress strain curves at different strain rates at the reference temperature $T_{r e f}=23^{\circ} \mathrm{C}$ (See figure 15) are used to identify the parameters for the yield stress, the isotropic hardening and the viscoplastic function, and only one stress-strain curve at different temperature than $T=23^{\circ} \mathrm{C}$, is used to calibrate the function $\Gamma(T)$, which is the one at the temperature $T=45^{\circ} \mathrm{C}$ and strain rate $\dot{\varepsilon}=9.310^{-6} \mathrm{~s}^{-1}$. The identified viscoplastic material parameters are listed in table 4 . The predicted stress strain curves at the temperatures $T=45^{\circ} \mathrm{C}$ and $T=60^{\circ} \mathrm{C}$ are compared to the experimental ones in figures 16 and 17 . Generally a good agreement can be seen between the experimental data and numerical predictions.

Yield stress: $\sigma_{y}(T)=\Gamma\left(\beta_{1}, T\right) \sigma_{y, r e f}$
$\quad T_{r e f}=23^{\circ} \mathrm{C}, \beta_{1}=0.018, \sigma_{y, r e f}=3.5 \mathrm{MPa}$

Isotropic hardening: $R(T, p)=\Gamma\left(\beta_{1}, T\right) k p^{n_{1}}\left(1-\exp \left(-n_{2} p\right)\right)$ $k=24 \mathrm{MPa}, n_{1}=0.18, n_{2}=230$

Viscoplastic function: $g_{v}=\frac{\sigma_{y}}{\eta}\left(\frac{f}{\sigma_{y}}\right)^{n}$ $\eta=1.2910^{6}$ MPa.s, $m=4.75$

Table 4: Viscoplastic parameters for PP 


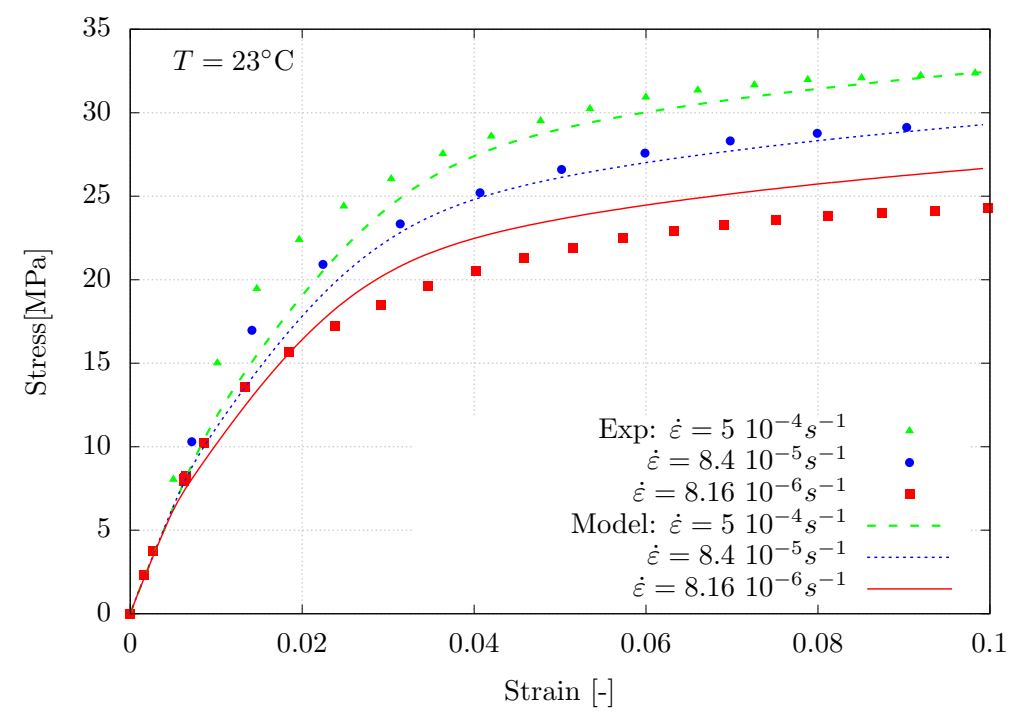

Figure 15: Tensile tests under different strain rates at temperature $T=23^{\circ} \mathrm{C}$

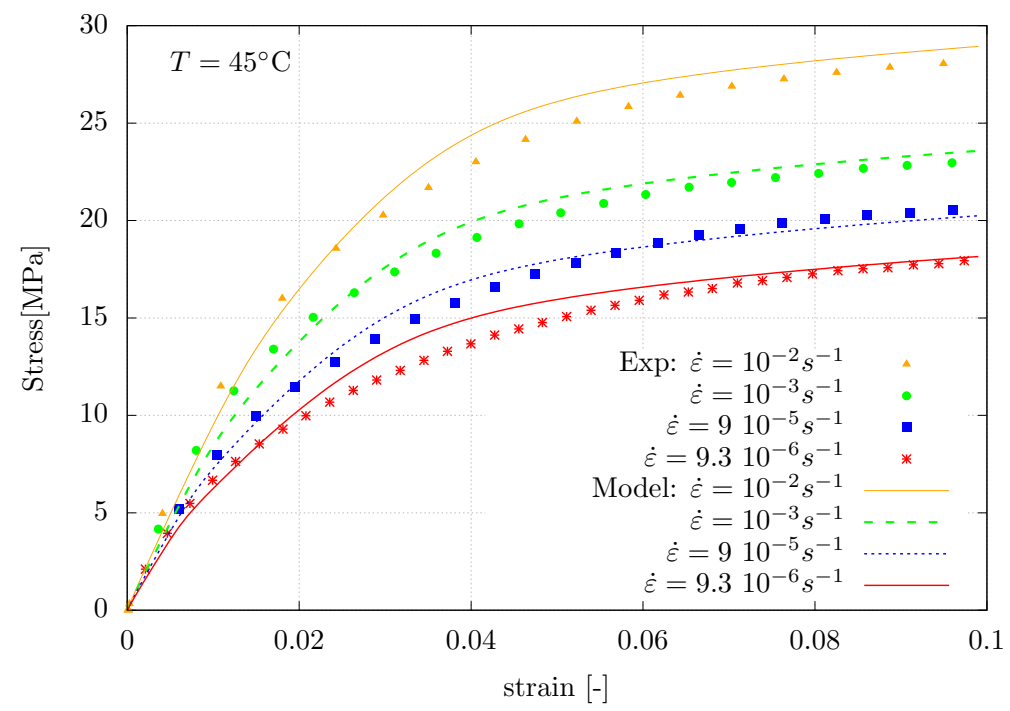

Figure 16: Tensile tests under different strain rates at temperature $T=45^{\circ} \mathrm{C}$ 


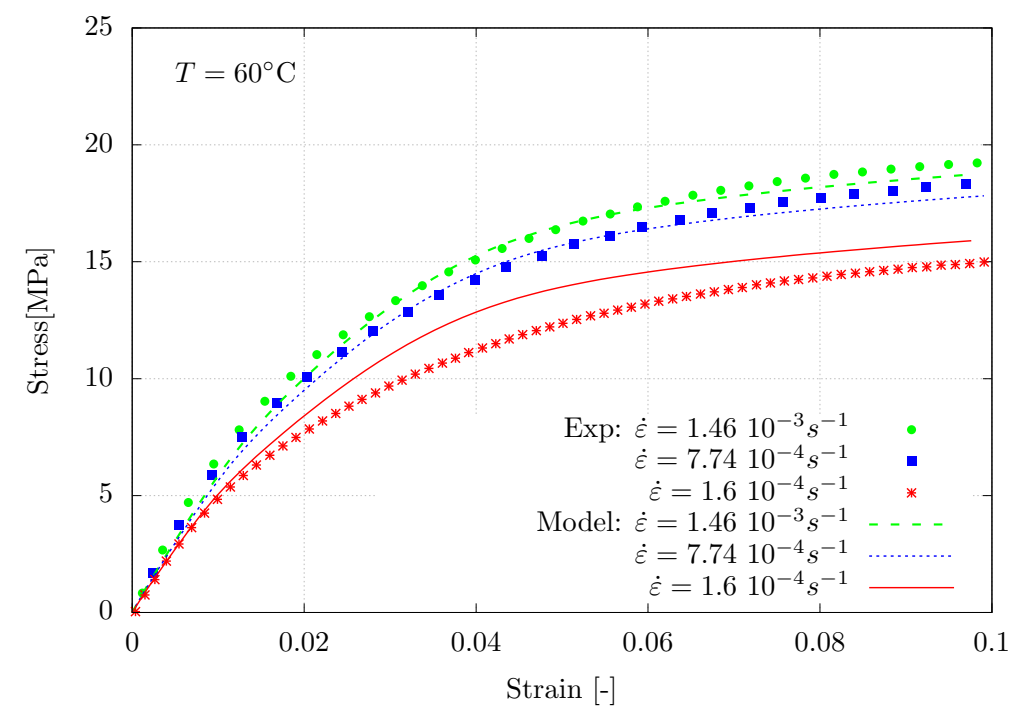

Figure 17: Tensile tests under different strain rates at temperature $T=60^{\circ} \mathrm{C}$

394 Similar to the case of PA66, the self heating of PP was estimated. The Figure 18 shows an example of the self-heating field within the dogbone specimen of $396 \mathrm{PP}$ under uniaxial test at $T=23^{\circ} \mathrm{C}$ and a strain rate $\dot{\varepsilon}=1.810^{-2} \mathrm{~s}^{-1}$. The predictions of the average surface temperature caused by self heating under 398 different strain rates at initial temperature of $T=23^{\circ} \mathrm{C}$ are plotted in figure 19 . 


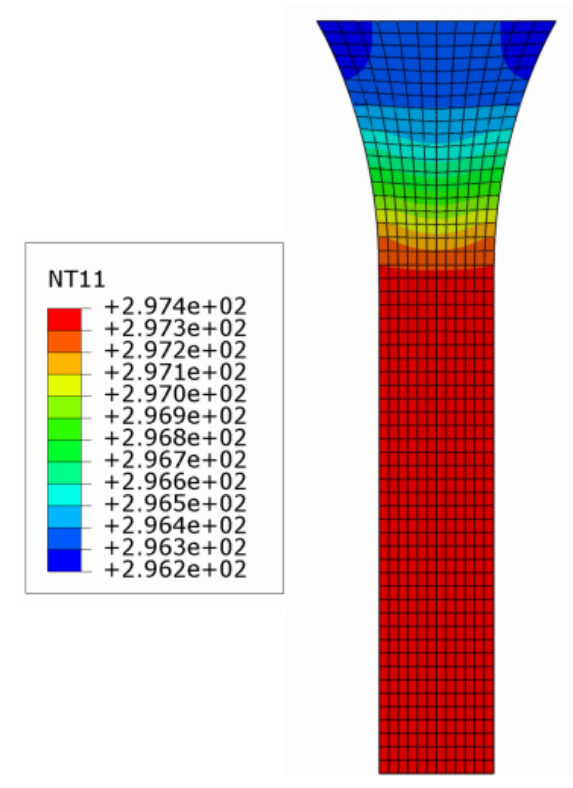

Figure 18: An example of the self-heating field within the dogbone specimen of PP under uniaxial test at $T=23^{\circ} \mathrm{C}$ and a strain rate $\dot{\varepsilon}=1.810^{-2} \mathrm{~s}^{-1}$. For the strain equal to 0.1 . The temperature values are in ${ }^{\circ} \mathrm{K}$.

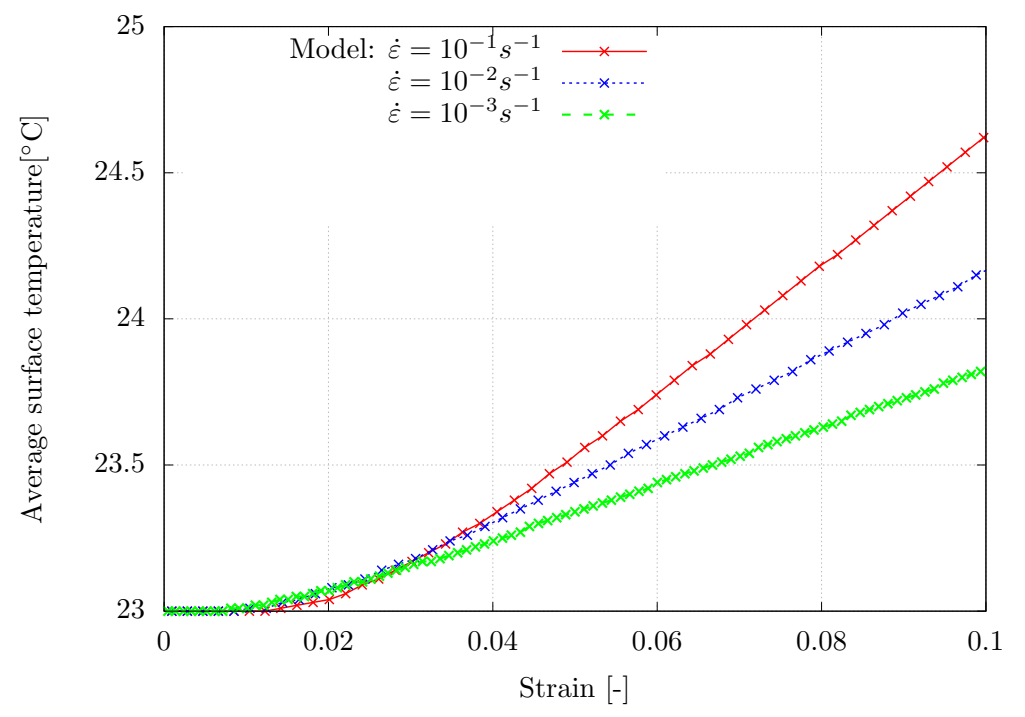

Figure 19: The predictions of the average surface temperature caused by self heating under different strain rates at initial temperature of $T=23^{\circ} \mathrm{C}$.

The proposed model was also employed to simulate the behavior of PP un400 der low cyclic fatigue loading. The results are compared to the experimental 
data of Shukla et al. (2014). The materials parameters already identified (for PP 575P from Sabic) were used in the following simulations. The performed fatigue tests by Shukla et al. (2014) are strain controlled tests. Specimens were injected following the standard ASTM D368 - Geometry type I. The specimens are subjected to displacement controlled sinusoidal loading at $10 \mathrm{HZ}$ with and amplitude of $0.2 \mathrm{~mm}$ or $0.35 \mathrm{~mm}$ and a mean displacement of $1 \mathrm{~mm}$. The tests are performed at room temperature $\left(R T=28^{\circ} \mathrm{C}\right)$. The force needed to keep the level of controlled strains, in the case of the amplitude equal to $0.2 \mathrm{~mm}$, during the loading is plotted in figure 20 . The temperature at the surface of sample was $4 \mathbf{1 0}$ measured experimentally using a non-contact type Raytek MI (REYMID10LT) temperature sensor. The simulated and measured temperatures are plotted in

${ }_{412}$ figure 21 . The temperature predictions are acceptable in the case of an amplitude equal to $0.2 \mathrm{~mm}$, however they are underestimated in the case of 0.35 ${ }_{414} \mathrm{~mm}$. Possible improvements of the model's predictive capabilities are discussed in section 4 .

416

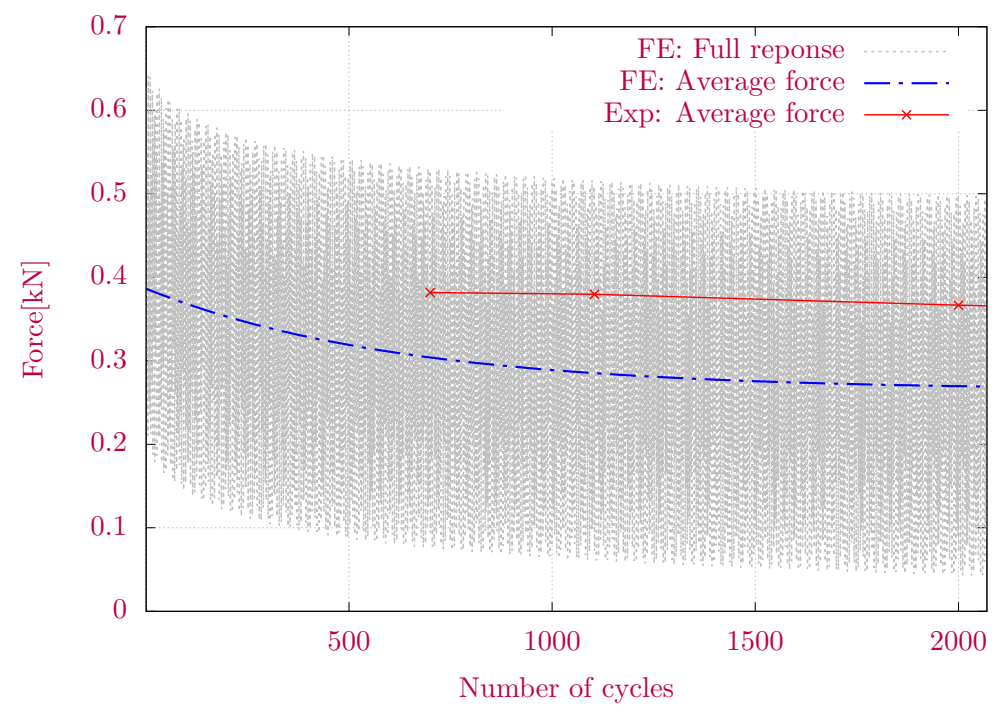

Figure 20: The average force variation with number of cycles for PP at initial temperature of $T=28^{\circ} \mathrm{C}$. 


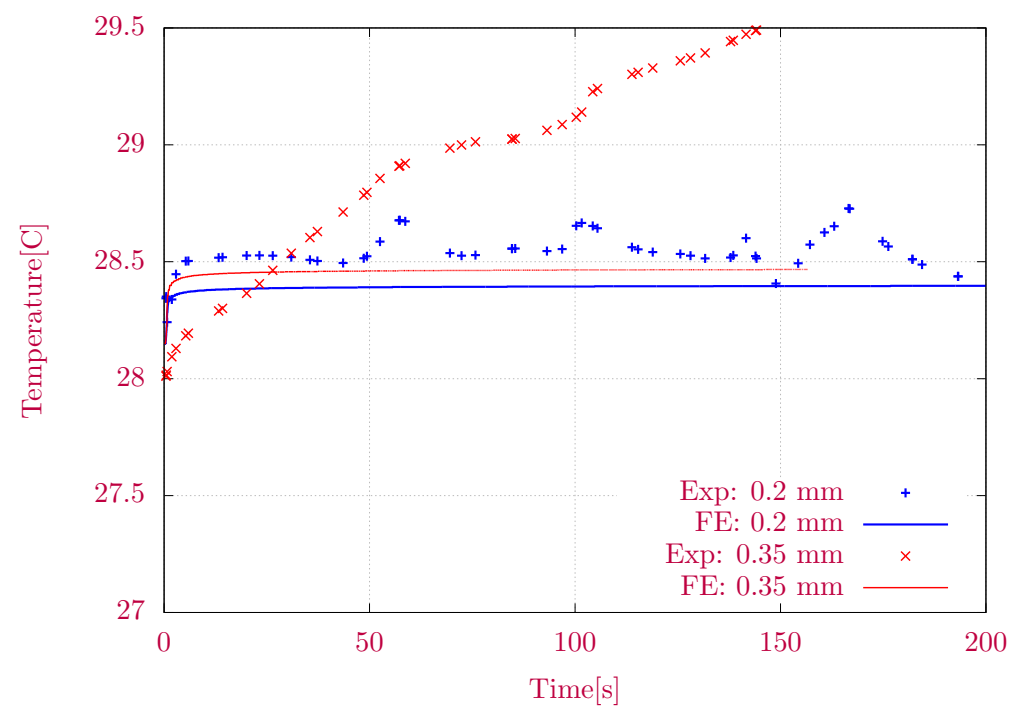

Figure 21: The surface temperature for PP specimens during the loading.

\section{4. Discussion}

We presented model a fully coupled thermo-viscoelastic and thermo-viscoplastic

420 model which allows to capture the effects of both loading rate and temperature on the behavior of thermoplastic polymers. The thermodynamical derivation al-

422 lows to obtain an estimation of the material self heating under different loading conditions. This effect may be the origin of material failure under specific load-

${ }_{\mathbf{4 2 4}}$ ing conditions. The model was validated against available experimental data on Nylon 66 (PA66) and PP under different loading conditions (i.e. tensile and shear loadings, monotonic and cyclic). The comparison between the predicted self heating and the experimental measurements showed an acceptable agreement in the case of PA66 and PP. Some improvements discussed hereafter can only enhance the predictive capabilities of the proposed model.

430 This work is restricted to the regime of small perturbations (small strains, displacements and rotations). An extension to the large deformation regime can be developed following the work of Gudimetla and Doghri (2017) who extended the small strain viscoelastic-viscoplastic model of Miled et al. (2011).

${ }_{434}$ The authors proposed an expression for the Helmholtz free energy defined as 
the sum of four contributions: viscoelastic, viscoplastic, softening and hyperelastic re-hardening. The viscoelastic part is defined by an extension of the work of Christensen and Freund (1971) to large deformations. For the other contributions, Gudimetla and Doghri (2017) took advantage of employed the huge knowledge about finite strain elasto(visco)plasticity which was developed mainly for metals, with special choices in term of strain and stress measures.

In the experimental validation section, The Poisson's ratio (PR) is assumed ${ }_{442}$ to be constant. However, this is a simplifying assumption because the PR for thermoplastic polymers, is proven to be time, stress and thermal expansion de-

444 pendent, which makes PR values determined from a uniaxial test not applicable to other uniaxial loadings with different time histories or to multi-axial loadings and thermal expansions. Rigorously, PR is not a material parameter for thermoplastics as already stated in Krairi and Doghri (2014). The use of shear and bulk time functions, expressed using Prony series determined directly from experiments is more appropriate to model the multi-axial aspects of material behavior. However, if the only available experimental data are uniaxial tests, then an estimate of the shear and bulk moduli may be found, assuming a constant 452 Poisson's ratio using expressions given in eq.(47).

Thermal properties mainly specific heating capacities, thermal conductivity ${ }_{454}$ and thermal expansion are proved to be temperature dependent experimentally. In the presented simulations those parameters are assumed to be constant. In the studied temperature range (lower than the melting temperature) small changes are expected. However further experimental investigations of the studied materials are needed to confirm this assumption.

From modeling point of view, sophisticated evolution laws needs to be developed, based on the material caloric behavior using accurate representation of material micro-structure characterized by its degree of crystallinity. Wunderlich (2003)

${ }_{462}$ showed that semi-crystalline polymers micro-structure consists of three phases: crystalline phase, mobile amorphous phase and rigid amorphous phase. Those

${ }_{464}$ phases exhibit changes in their densities and concentrations because thermomechanical histories of loading.

466 As already mentioned in the introduction taking into account the material micro-structure may be performed using micro-mechanical models such as Nikolov 
468

472

476

478

480

482

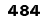

490 (e.g. Gudimetla and Doghri, 2017).

494 sure, several authors proposed the use of a notched bar (e.g. Cayzac et al., 2013b; Laiarinandrasana et al., 2016; Ognedal et al., 2014). According to these authors a triaxial stress sate dominated by hydrostatic pressure is expected near the notch. A so called plastic delation is observed by the authors for HDPE and PVC (Ognedal et al., 2014), and for PA6 (Laiarinandrasana et al., 2016).

$\mathbf{5 0 0}$ Microscopic investigations showed that this deformation is found to be related

2016; Lion et al., 2017). In the presented approach the effect of these microstructural changes are modeled in a macroscopic phenomenological way by using thermo-viscoelasticity employing the TTS principle (via WLF function) and thermo-viscoplasticity with temperature sensitivity function.

For some thermoplastic polymers, it is important to take into account the hydrostatic pressure sensitivity. This sensitivity may be characterized by several methods, for example by comparing the material response in terms of absolute values of true stress and true strain under uniaxial tension and uniaxial compression. Maurel-Pantel et al. (2015) assumed that PA66 is incompressible since the the uniaxial tensile tests are not enough to judge about the pressure sensitivity of the material. A complete multi-axial testing campaign is needed in order to check this sensitivity, an example of this study is the work of Farrokh and Khan (2010) who studied the multi-axial behavior of Nylon 101, under several loading conditions: tension, compression, tension-torsion, torsion, biaxial compression and reverse torsion. The authors were able to construct an experimental yield surfaces at different strain rates, whose shape should show the pressure sensimulti-axial behavior of the studied polymer, the proposed model may be used. Otherwise, plastic deformation may occur due to hydrostatic pressure which is not captured by the proposed model. In fact, using von Mises yield criterion makes the viscoplastic behavior insensitive to hydrostatic pressure, but the thermo-viscoelastic response should be influenced. Possible enhancement may be achieved by using a pressure sensitive yield criterion such as Drucker-Prager

As an experimental method to better capture the influence of hydrostatic pres- 
to the creation of voids inside the materials during loading and it is related to material softening and damage. From a modeling point of view, mechanical damage (or softening) may be taken into account by two different approaches.

504 The first approach is based on micromechanics, e.g. Gurson's model (Gurson, 1975, 1977). The second one is continuum damage mechanics (CDM) (e.g.

506 Lemaitre and Chaboche, 1978; Lemaitre, 1992); (a list of works employing the different approaches may be found in (Krairi and Doghri, 2014). Using CDM may allow to capture the effect of hydrostatic pressure implicitly on the material behavior, through the damage evolution laws. The present model can be extended in order to take the damage effect following the approach proposed in Krairi and Doghri (2014).

512 Actually, it is expected that better predictions of self-heating under cyclic loadings can be achieved by making the viscoelastic response nonlinear instead of

$\mathbf{5 1 4}$ linear in the present model. Indeed, in the isothermal case, Krairi and Doghri (2014) rendered the viscoelastic response nonlinear by coupling it to a damage model, and obtained better predictions of hysteresis loops under cyclic loadings than with other models. Other nonlinear viscoelastic formulations exist (e.g.

518 Schapery, 1967).

The proposed approach allows to model thermal softening. In the following, a notched round bar is studied under uniaxial tensile loading with different displacement rates. The presence of hydrostatic pressure is expected to increase

$\mathbf{5 2 2}$ self heating compared to our model's predicted temperature increase. Experimental investigations are needed to check this assumption. The bar geometry is

524 taken from Laiarinandrasana et al. (2016) cf. figure 22-(a). In order to show the effect of the notch on notched bar response, the case of unnotched sample (the same geometry without a notch) is also studied. Based on the axi-symmetry of the sample, one fourth was used in the FEM simulation (cf. figure 22-(b) and

$\left.{ }_{528}(\mathrm{c})\right)$. The bars are assumed to be made with PA66 and the material parameters identified in the previous section are used. Controlled displacement tensile tests

530 at different displacement rates of the unnotched and notched bars are simulated. The figure 23 shows the triaxiality ratio in the case of notched and unnotched

532 bars at their mid-sections. The triaxiality ratio is defined as the ratio between the hydrostatic pressure and the von Mises equivalent stress $\left(\boldsymbol{\sigma}_{H} / \sigma_{e q}\right)$. It allows 
534 to quantify the contribution of hydrostatic pressure to the stress state. It can be seen from figure 23 that for the unnotched bar the triaxiality is constant

536 and rate independent, and it value is lower than the triaxiality in the case of notched bar. For the notched bar the triaxiality near the center of the bar midsection is higher than the one near the notch and its value is increasing with the increase of applied displacement. In contrast to the case of unnotched bar,

540 the triaxiality for notched bar is rate dependent. The presence of the notch caused a stress concentration and a hydrostatic pressure dominated stress state,

$\mathbf{5 4 2}$ which influences the overall response of the notched samples in terms of reaction forces and generated temperature under increasing applied displacement with

544 different rates. The figures 24 and 25 show the reactions forces versus the applied displacement, respectively for unnotched and notched bars. A drop of the

546 force can be noticed for the case of notched bar, which is explained by thermal softening due to high self heating near the notch as showed in figure 26. The

548 values of self-heating in the case of unnotched bars at their external surface are plotter in figure 27. Compared to notched bar case, these values are small and 550 are not influencing the bar response as in the case of notched bars.

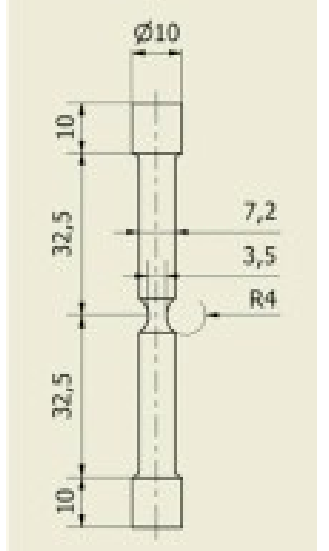

(a)

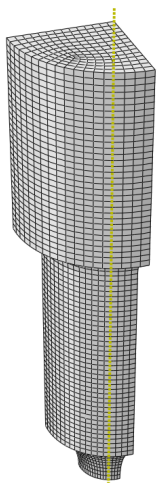

(b)

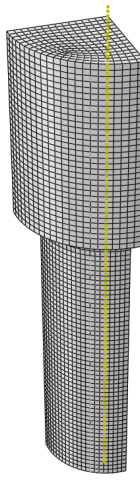

(c)

Figure 22: (a) The dimensions of the notched bar from Laiarinandrasana et al. (2016), (b) The employed mesh for the FE simulations on Notched bar, (c) The employed mesh for the FE simulations on Unnotched bar 


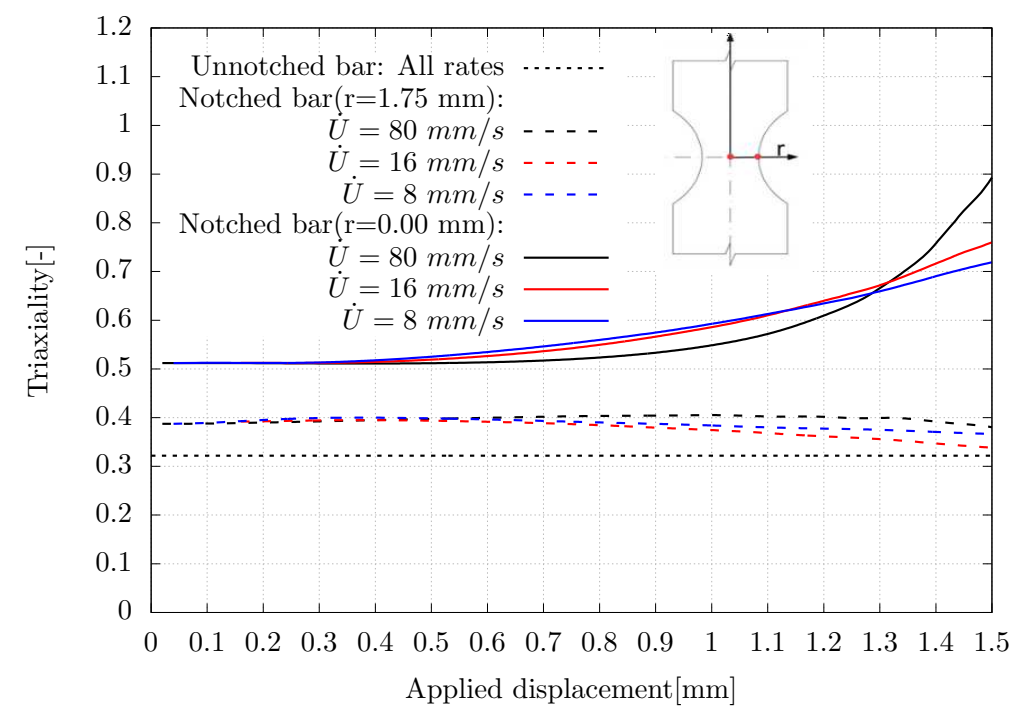

Figure 23: Unnotched and Notched PA66 bar. Triaxiality versus applied displacement curves with different displacement rates.

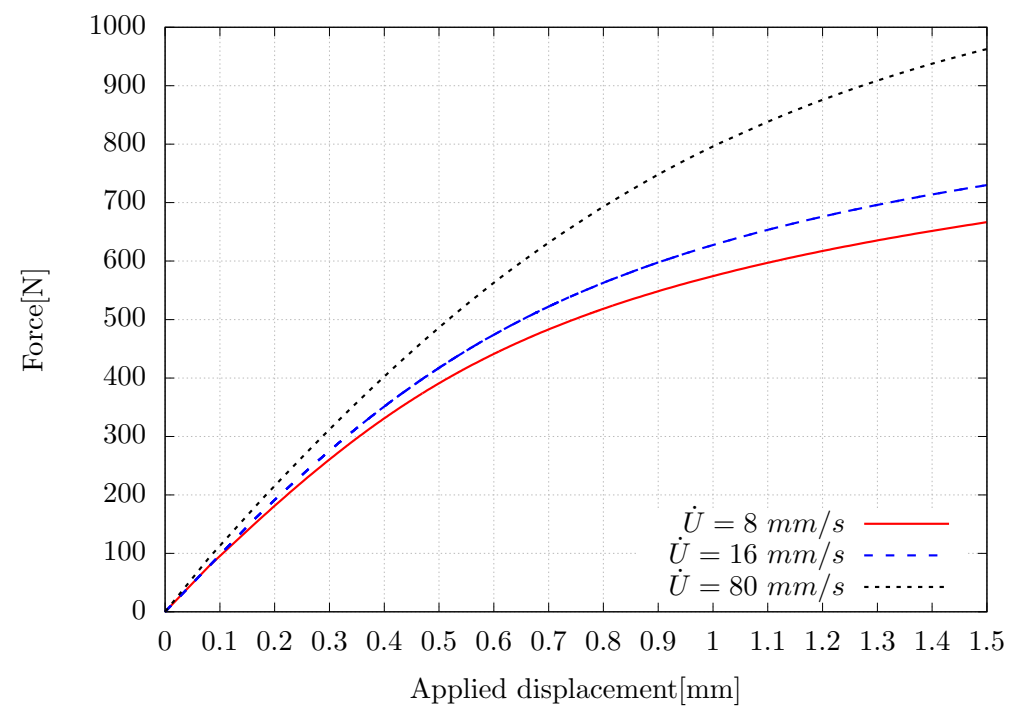

Figure 24: Unnotched PA66 bar. Predicted force versus applied displacement curves with different displacement rates. 


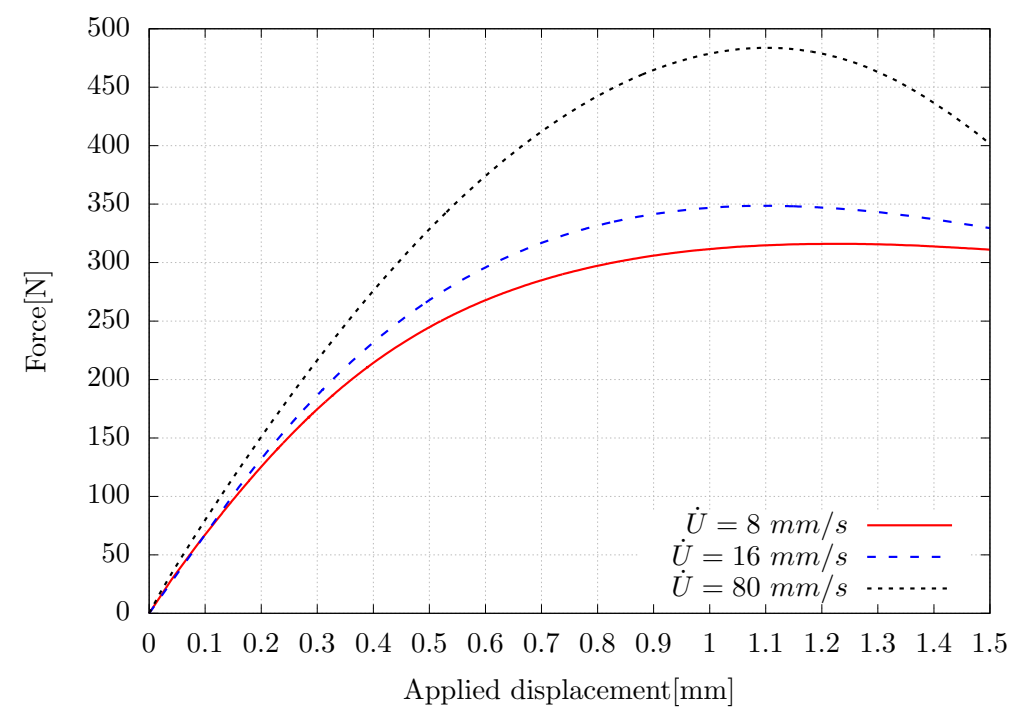

Figure 25: Notched PA66 bar. Predicted force versus applied displacement curves with different displacement rates.

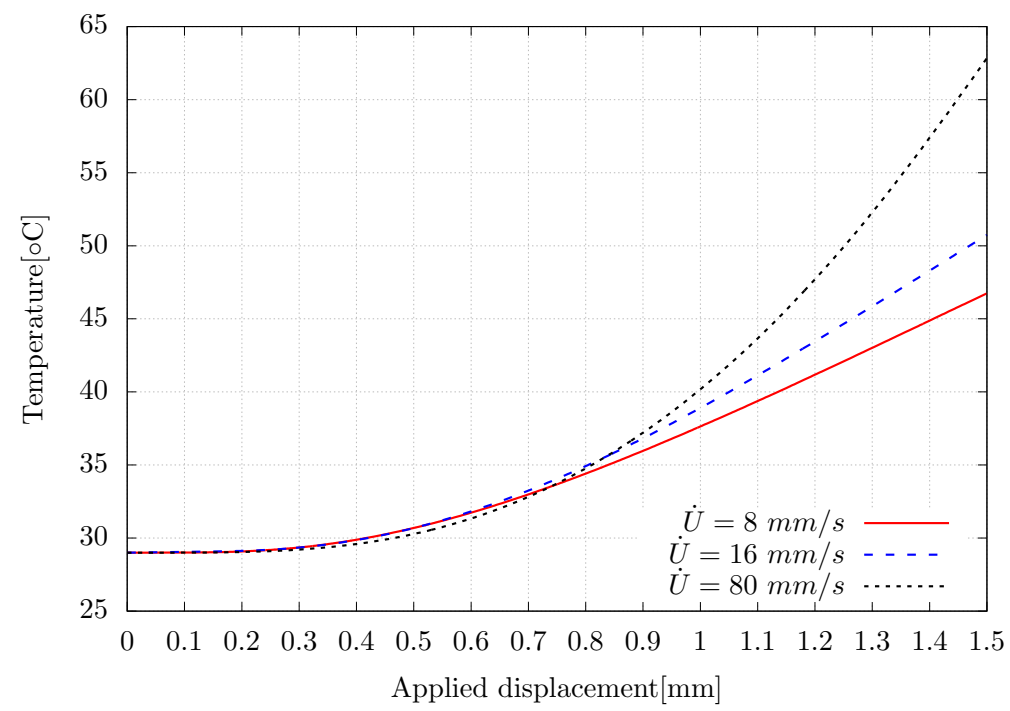

Figure 26: Notched PA66 bar. Predicted temperature near the notch versus applied displacement, for different displacement rates. 


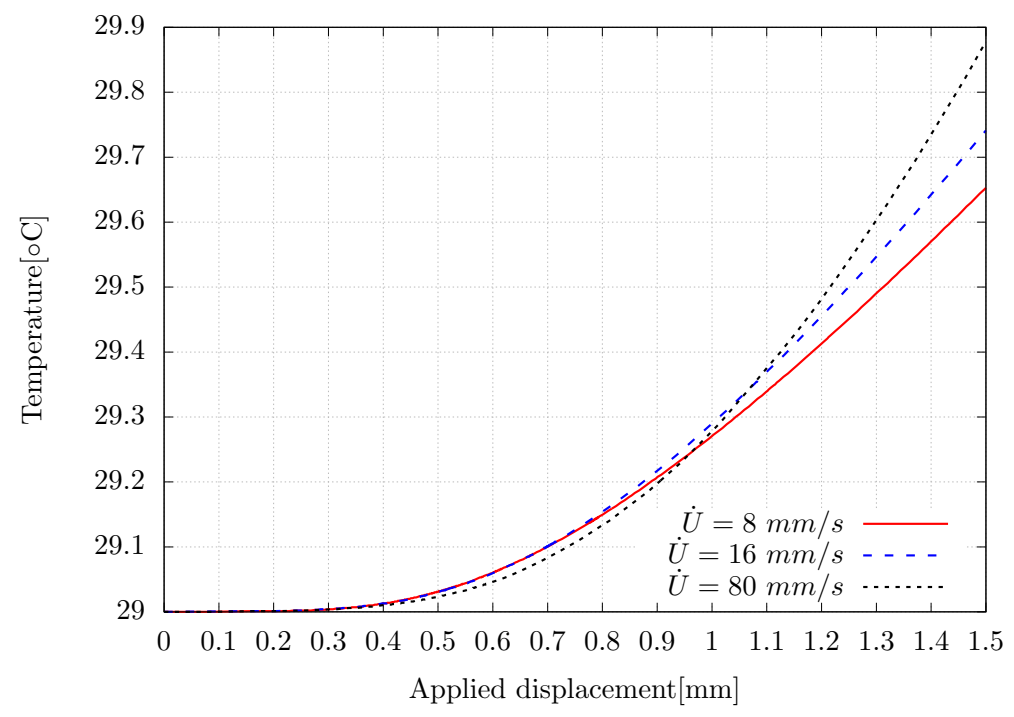

Figure 27: Unotched PA66 bar. Predicted temperature near the notch versus applied displacement, for different displacement rates.

\section{Conclusions}

558

560 stress.

568 The experimental validation using uniaxial and shear tests at different strain rates and different temperature on Polyamide 66 (PA66) and Polypropylene

570 (PP) showed that the model captures successfully the rate dependency and temperature sensitivity of the polymer materials. Possible enhancement and $\mathbf{5 7 2}$ improvement of the proposed approach are presented in the discussion section. 


\section{Acknowledgments}

574 This work has been undertaken partially in the framework of the strategic basic project VLAIO SBO-150013 project Composite Heat Exchangers (www.compohex.ugent.be)

576 funded by Flanders Innovation \& Entrepreneurship (VLAIO). It has been also undertaken partially in the framework of the DURAFIP project funded by

578 France under the FUI (Fonds unique interministériel) and the Walloon Region under the EUREKA programme (convention 1117479).

\section{AppendixA. Thermodynamical derivation of constitutive relations}

We make a unified presentation for the two cases when viscoelastic properties 582 are temperature independent or dependent. In the former case, it suffices to take $\bar{t}=t$ and $a_{T}=1$ in the following equations, while in the latter, $\bar{t}$ and $a_{T}$ do

584 represent the reduced time and the time shift function, respectively

The proposed Helmholtz free energy function $\psi$ is defined as:

$$
\psi=\psi^{v e}+\psi^{h}
$$

586 the following decomposition $\varepsilon=\varepsilon^{v e}+\varepsilon^{t h}+\varepsilon^{v p}$ where $\varepsilon$ is the total strain, $\varepsilon^{v p}$ the viscoplastic strain and the thermo-viscoelastic strain $\left(\varepsilon^{t v e}=\varepsilon^{t h}+\varepsilon^{v e}\right)$. In the following, due to the hypothesis of small perturbation, we have $\frac{d}{d t}=\frac{\partial}{\partial t}$ and similarly to Christensen (1982) in pure viscoelasticity, the expressions of the Cauchy stress and the entropy are found using the inequality of ClausiusDuhem which requires the dissipation to be non-negative

$$
\phi=\boldsymbol{\sigma}: \dot{\varepsilon}-\rho(\dot{\psi}+S \dot{T})-\nabla T \cdot \frac{\boldsymbol{q}}{T} \geq 0
$$

Applying the Leibnitz rule to time derivative of the free energy equations (3) to 
(6) . the Clausius-Duhem inequality can be re-expressed as:

$$
\begin{aligned}
& \left\{\boldsymbol{\sigma}(t)-\boldsymbol{D}(0)-\int_{-\infty}^{t} \mathbb{C}^{v e}(\bar{t}-\bar{\tau}, 0): \frac{\partial \boldsymbol{\varepsilon}^{t v e}(\tau)}{\partial \tau} d \tau+\int_{-\infty}^{t} \boldsymbol{\varphi}(0, \bar{t}-\bar{\tau}) \frac{\partial \theta(\tau)}{\partial \tau} d \tau\right\}: \dot{\boldsymbol{\varepsilon}}^{t v e}(t) \\
& +\left\{-\rho S(t)+\beta(0)+\int_{-\infty}^{t} m(\bar{t}-\bar{\tau}, 0) \frac{\partial \theta(\tau)}{\partial \tau} d \tau+\int_{-\infty}^{t} \boldsymbol{\varphi}(\bar{t}-\bar{\tau}, 0): \frac{\partial \varepsilon^{t v e}(\tau)}{\partial \tau} d \tau\right\} \dot{\theta}(t) \\
& -\int_{-\infty}^{t} \frac{\partial}{\partial t} D(\bar{t}-\bar{\tau}): \frac{\partial \varepsilon^{t v e}(\tau)}{\partial \tau} d \tau+\int_{-\infty}^{t} \frac{\partial}{\partial t} \beta(\bar{t}-\bar{\tau}) \frac{\partial \theta(\tau)}{\partial \tau} d \tau+\Lambda \\
& +\boldsymbol{\sigma}: \dot{\boldsymbol{\varepsilon}}^{v p}-R(r) \frac{d p(t)}{d t}-a \boldsymbol{\chi}(t): \frac{d \boldsymbol{\chi}(t)}{d t}-\nabla T \cdot \frac{\boldsymbol{q}}{T} \geq 0
\end{aligned}
$$

Where $\Lambda$ is the dissipation term due to the time dependence of the relaxation

594 functions and is given by:

$$
\begin{aligned}
\Lambda= & -\frac{1}{2} \int_{-\infty}^{t} \int_{-\infty}^{t} \frac{\partial \varepsilon^{t v e}(\tau)}{\partial \tau}: \frac{\partial \mathbb{C}^{v e}(\bar{t}-\bar{\tau}, \bar{t}-\bar{\eta})}{\partial t}: \frac{\partial \varepsilon^{t v e}(\eta)}{\partial \eta} d \tau d \eta \\
& +\int_{-\infty}^{t} \int_{-\infty}^{t} \frac{\partial \varphi(\bar{t}-\bar{\tau}, \bar{t}-\bar{\eta})}{\partial t}: \frac{\partial \varepsilon^{t v e}(\eta)}{\partial \eta} \frac{\partial \theta(\tau)}{\partial \tau} d \eta d \tau \\
& +\frac{1}{2} \int_{-\infty}^{t} \int_{-\infty}^{t} \frac{\partial m(\bar{t}-\bar{\tau}, \bar{t}-\bar{\eta})}{\partial t} \frac{\partial \theta(\eta)}{\partial \eta} \frac{\partial \theta(\tau)}{\partial \tau} d \eta d \tau
\end{aligned}
$$

The following symmetry properties (Christensen and Freund, 1971; Christensen, 596 1982) and particular forms are used

$$
\begin{aligned}
\mathbb{C}_{i j k l}^{v e}(\tau, \eta) & =\mathbb{C}_{k l i j}^{v e}(\eta, \tau)=\mathbb{C}_{k l i j}^{v e}(\eta+\tau) \\
\boldsymbol{\varphi}_{i j}(\tau, \eta) & =\boldsymbol{\varphi}_{j i}(\eta, \tau)=\boldsymbol{\varphi}_{i j}(\eta+\tau) \\
m(\tau, \eta) & =m(\eta, \tau)=m(\eta+\tau)
\end{aligned}
$$

The inequality A.3 must hold for all arbitrary values of the derivatives $\dot{\varepsilon}^{t v e}(t)$ and $\dot{\theta}(t)$. Therefore their coefficients should vanish. Hence,

$$
\begin{gathered}
\boldsymbol{\sigma}(t)=\boldsymbol{D}(0)+\int_{-\infty}^{t} \mathbb{C}^{v e}(\bar{t}-\bar{\tau}): \frac{\partial \varepsilon^{t v e}(\tau)}{\partial \tau} d \tau-\int_{-\infty}^{t} \boldsymbol{\varphi}(\bar{t}-\bar{\tau}) \frac{\partial \theta(\tau)}{\partial \tau} d \tau \\
\rho S(t)=\beta(0)+\int_{-\infty}^{t} m(\bar{t}-\bar{\tau}) \frac{\partial \theta(\tau)}{\partial \tau} d \tau+\int_{-\infty}^{t} \boldsymbol{\varphi}(\bar{t}-\bar{\tau}): \frac{\partial \varepsilon^{t v e}(\tau)}{\partial \tau} d \tau
\end{gathered}
$$


The dissipation is then expressed as

$$
\begin{aligned}
\phi= & -\int_{-\infty}^{t} \frac{\partial}{\partial t} D(\bar{t}-\bar{\tau}): \frac{\partial \varepsilon^{t v e}(\tau)}{\partial \tau} d \tau+\int_{-\infty}^{t} \frac{\partial}{\partial t} \beta(\bar{t}-\bar{\tau}) \frac{\partial \theta(\tau)}{\partial \tau} d \tau \\
& +\Lambda-\nabla T \cdot \frac{\boldsymbol{q}}{T}+\boldsymbol{\sigma}: \dot{\boldsymbol{\varepsilon}}^{v p}-R \dot{p}-a \boldsymbol{\chi}(t): \dot{\boldsymbol{\chi}}(t) \geq 0
\end{aligned}
$$

$\boldsymbol{D}(0)$ and $\beta(0)$ are the initial stress and initial entropy, respectively, and they 598 are assumed to be null. According to Christensen (1982) in order to satisfy the inequality A.8 for all processes, it is necessary that:

$$
\frac{\partial}{\partial t} D(\bar{t})=0, \quad \frac{\partial}{\partial t} \beta(\bar{t})=0
$$

consequently

$$
\phi=-\nabla T \cdot \frac{\boldsymbol{q}}{T}+\boldsymbol{\sigma}: \dot{\boldsymbol{\varepsilon}}^{v p}-R \dot{p}-a \boldsymbol{\chi}(t): \dot{\boldsymbol{\chi}}(t) \geq 0
$$

600 where $\Lambda$ being a second order term, it must be neglected. In equation A.6, if we replace $\varepsilon^{t v e}$ by its expression $\left(\varepsilon^{t v e}=\varepsilon^{t h}+\varepsilon^{v e}\right)$ then

$$
\boldsymbol{\sigma}(t)=\int_{-\infty}^{t}\left\{\mathbb{C}^{v e}(\bar{t}-\bar{\tau}):\left(\frac{\partial \varepsilon^{v e}(\tau)}{\partial \tau}+\frac{\partial(\boldsymbol{\alpha} \theta(\tau))}{\partial \tau}\right)-\boldsymbol{\varphi}(\bar{t}-\bar{\tau}) \frac{\partial \theta(\tau)}{\partial \tau}\right\} d \tau
$$

602 In the isotropic case, $\varphi$ can be expressed as $\varphi_{i j}=\varphi(t) \delta_{i j}$. After replacing the relaxation tensor by its expression (cf. equation 25 ). The Cauchy stress may be divided into deviatoric and hydrostatic stresses:

$$
\boldsymbol{\sigma}(t)=\boldsymbol{s}(t)+\sigma_{H}(t) \mathbf{1}
$$

The viscoelastic strain tensor may be also divided into deviatoric and dilata606 tional parts:

$$
\varepsilon^{v e}(t)=\boldsymbol{\xi}^{v e}(t)+\epsilon_{H}^{v e}(t) \mathbf{1}
$$


Consequently,

$$
\left\{\begin{aligned}
& s_{i j}(t)= \int_{-\infty}^{t} 2 G(\bar{t}-\bar{\tau}) \frac{\partial \boldsymbol{\xi}_{i j}^{v e}(\tau)}{\partial \tau} d \tau \\
& \sigma_{H}(t)=\int_{-\infty}^{t}\left\{3 K(\bar{t}-\bar{\tau})\left(\frac{\partial \epsilon_{H}^{v e}(\tau)}{\partial \tau}+\alpha(\tau) \frac{\partial \theta(\tau)}{\partial \tau}+\frac{\partial \alpha(\tau)}{\partial \tau} \theta(\tau)\right)\right. \\
&\left.-\varphi(\bar{t}-\bar{\tau}) \frac{\partial \theta(\tau)}{\partial \tau}\right\} d \tau
\end{aligned}\right.
$$

608 We assume that the absolute value of $\frac{\partial \alpha(\tau)}{\partial \tau} \theta(\tau)$ is negligible in front of the absolute value of $\alpha(\tau) \frac{\partial \theta(\tau)}{\partial \tau}$. In order to retrieve familiar expressions by ${ }_{610}$ choosing $\varphi(t)=3 \alpha K(\bar{t})$, the final expressions of the stress is

$$
\left\{\begin{array}{l}
s_{i j}(t)=2 \int_{-\infty}^{t} G(\bar{t}-\bar{\tau}) \frac{\partial \boldsymbol{\xi}_{i j}^{v e}(\tau)}{\partial \tau} d \tau \\
\sigma_{H}(t)=3 \int_{-\infty}^{t} K(\bar{t}-\bar{\tau}) \frac{\partial \epsilon_{H}^{v e}(\tau)}{\partial \tau} d \tau
\end{array}\right.
$$

These expressions are equivalent to:

$$
\boldsymbol{\sigma}(t)=\int_{-\infty}^{t} \mathbb{C}^{v e}(\bar{t}-\bar{\tau}): \frac{\partial \varepsilon^{v e}(\tau)}{\partial \tau} d \tau
$$

${ }_{612}$ The final expression of the entropy is

$$
\rho S(t)=\int_{-\infty}^{t}\left(m(\bar{t}-\bar{\tau})+9 \alpha^{2} K(\bar{t}-\bar{\tau})\right) \frac{\partial \theta(\tau)}{\partial \tau} d \tau
$$




\section{References}

Balieu, R., Lauro, F., Bennani, B., Delille, R., Matsumoto, T., Mottola, E., 2013.

Alisafaei, F., Han, C.-S., Garg, N., 2016. On couple-stress elasto-plastic constitutive frameworks for glassy polymers. International Journal of Plasticity 77, 30-53.

Ames, N. M., Srivastava, V., Chester, S. A., Anand, L., Aug. 2009. A thermomechanically coupled theory for large deformations of amorphous polymers. Part II: Applications. International Journal of Plasticity 25 (8), 1495-1539.

URL http://www.sciencedirect.com/science/article/pii/S074964190800171X

Anand, L., Ames, N. M., Srivastava, V., Chester, S. A., Aug. 2009. A thermomechanically coupled theory for large deformations of amorphous polymers. Part I: Formulation. International Journal of Plasticity 25 (8), 1474-1494.

URL http://www.sciencedirect.com/science/article/pii/S0749641908001708

Anand, L., Gurtin, M. E., Mar. 2003. A theory of amorphous solids undergoing large deformations, with application to polymeric glasses. International Journal of Solids and Structures 40 (6), 1465-1487.

URL http://www.sciencedirect.com/science/article/pii/S0020768302006510

Arruda, E. M., Boyce, M. C., Jayachandran, R., Jan. 1995. Effects of strain rate, temperature and thermomechanical coupling on the finite strain deformation of glassy polymers. Mechanics of Materials 19 (2), 193-212.

URL http://www.sciencedirect.com/science/article/pii/016766369400034E

Ayoub, G., Za $\tilde{A}^{-}$ri, F., FrÃ@derix, C., Gloaguen, J. M., Na $\tilde{A}^{-}$t-Abdelaziz, M., Seguela, R., Lefebvre, J. M., Apr. 2011. Effects of crystal content on the mechanical behaviour of polyethylene under finite strains: Experiments and constitutive modelling. International Journal of Plasticity 27 (4), 492-511.

URL http://www.sciencedirect.com/science/article/pii/S0749641910000963

A fully coupled elastoviscoplastic damage model at finite strains for mineral filled semi-crystalline polymer. International Journal of Plasticity.

Ball, R. C., Doi, M., Edwards, S. F., Warner, M., Aug. 1981. Elasticity of entangled networks. Polymer 22 (8), 1010-1018.

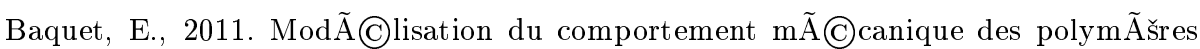
644 semi-cristallins. Application au PA66 sous chargement complexe et non monotone. Ph.D. thesis, CEMEF, Ecole des Mines de Paris, France. 
Billon, N., 2012. New constitutive modeling for time-dependent mechanical behavior of polymers close to glass transition: Fundamentals and experimental validation. Journal of Applied Polymer Science 125 (6), 4390-4401.

Bouvard, J. L., Ward, D. K., Hossain, D., Nouranian, S., Marin, E. B., Horstemeyer, M. F., Sep. 2009. Review of Hierarchical Multiscale Modeling to Describe the Mechanical Behavior of Amorphous Polymers. Journal of Engineering Materials and Technology 131 (4), 041206-041206-15. URL http://dx.doi.org/10.1115/1.3183779

Boyce, M. C., Parks, D. M., Argon, A. S., 1988. Large inelastic deformation of glassy polymers. Part I: rate dependent constitutive model. Mechanics of Materials 7 (1), 15-33.

664

Buckley, C. P., Jones, D. C., Jan. 1995. Glass-rubber constitutive model for amorphous polymers near the glass transition. Polymer 36 (17), 3301-3312.

666 URL http://www.sciencedirect.com/science/article/pii/003238619599429X

Cayzac, H.-A., Sa $\widetilde{A}^{-}$, K., Laiarinandrasana, L., 2013a. Damage based constitutive relationships in semi-crystalline polymer by using multi-mechanisms model. International Journal of Plasticity 51, 47-64.

Cayzac, H.-A., Saï, K., Laiarinandrasana, L., 2013b. Damage based constitutive relationships in semi-crystalline polymer by using multi-mechanisms model. International Journal of Plasticity 51, 47-64.

Chaboche, J.-L., 1997. Thermodynamic formulation of constitutive equations and application to the viscoplasticity and viscoelasticity of metals and polymers. International Journal of Solids and Structures 34 (18), 2239 - 2254.

Christensen, R., 1982. Theory of viscoelasticity: an introduction. Access Online via Elsevier. 
678

680

Dasari, A., Misra, R. D. K., Oct. 2003. On the strain rate sensitivity of high density

Doghri, I., 1993. Fully implicit integration and consistent tangent modulus in elasto-

Christensen, R. M., Freund, L., 1971. Theory of viscoelasticity. Journal of Applied Mechanics 38, 720 .

Christensen, R. M., Naghdi, P. M., 1967. Linear non-isothermal viscoelastic solids. Acta Mechanica $3(1), 1-12$.

Colak, O. U., Jan. 2005. Modeling deformation behavior of polymers with viscoplasticity theory based on overstress. International Journal of Plasticity 21 (1), 145-160.

URL http://www.sciencedirect.com/science/article/pii/S074964190400049X polyethylene and polypropylenes. Materials Science and Engineering: A 358 (1), $356-371$.

URL http://www.sciencedirect.com/science/article/pii/S0921509303003307 plasticity. International journal for numerical methods in engineering 36 (22), 39153932.

Doghri, I., 2000. Mechanics of deformable solids: linear, nonlinear, analytical and computational aspects. Springer Science \& Business Media.

694

Drozdov, A., 2011. Cyclic strengthening of polypropylene under strain-controlled loading. Materials Science and Engineering: A 528 (29), 8781-8789.

Drozdov, A., Christiansen, J. d., 2007. Cyclic viscoplasticity of high-density polyethylene: experiments and modeling. Computational materials science 39 (2), 465-480.

Dusunceli, N., Colak, O. U., 2008. Modelling effects of degree of crystallinity on mechanical behavior of semicrystalline polymers. International Journal of Plasticity 24 (7), 1224-1242.

Edwards, S. F., Vilgis, T., Apr. 1986. The effect of entanglements in rubber elasticity. Polymer 27 (4), 483-492.

URL http://www.sciencedirect.com/science/article/pii/0032386186902314

Farrokh, B., Khan, A. S., 2010. A strain rate dependent yield criterion for isotropic polymers: low to high rates of loading. European Journal of Mechanics-A/Solids 29 (2), 274-282.

Frank, G. J., Brockman, R. A., 2001. A viscoelastic-viscoplastic constitutive model for 708 glassy polymers. International Journal of Solids and Structures 38 (30), 5149-5164. 
Garcia-Gonzalez, D., Zaera, R., Arias, A., 2017. A hyperelastic-thermoviscoplastic con710 stitutive model for semi-crystalline polymers: Application to peek under dynamic loading conditions. International Journal of Plasticity 88, 27-52.

Ghorbel, E., 2008. A viscoplastic constitutive model for polymeric materials. International Journal of Plasticity 24 (11), 2032-2058.

Govaert, L., Timmermans, P., Brekelmans, W., 2000. The influence of intrinsic strain softening on strain localization in polycarbonate: modeling and experimental validation. Journal of Engineering Materials and Technology 122 (2), 177-185.

Gudimetla, M. R., Doghri, I., 2017. A finite strain thermodynamically-based constitu-

718

720

Gueguen, O., Richeton, J., Ahzi, S., Makradi, A., Apr. 2008. Micromechanically based formulation of the cooperative model for the yield behavior of semi-crystalline polymers. Acta Materialia 56 (7), 1650-1655.

724 URL http://www.sciencedirect.com/science/article/pii/S1359645407008488

Gurson, A., 1977. Continum theory of ductile rupture by void nucleation and growth: 726 Part I. Yield criterion and flow rules for porous materials. J. Eng. Mater. Technol 2.

Gurson, A. L., 1975. Continuum theory of ductile rupture by void nucleation and growth. Part I. Yield criteria and flow rules for porous ductile media. Tech. rep., Brown Univ., Providence, RI (USA). Div. of Engineering.

Hasan, O., Boyce, M., 1995. A constitutive model for the nonlinear viscoelastic viscoplastic behavior of glassy polymers. Polymer Engineering \& Science 35 (4), 331344.

Haward, R. N., Thackray, G., Jan. 1968. The use of a mathematical model to describe isothermal stress-strain curves in glassy thermoplastics. Proc. R. Soc. Lond.

736 A 302 (1471), 453-472.

URL http://rspa.royalsocietypublishing.org/content/302/1471/453

738 Kennedy, M., Peacock, A., Mandelkern, L., 1994. Tensile properties of crystalline polymers: linear polyethylene. Macromolecules 27 (19), 5297-5310. 
Khan, A. S., Farrokh, B., 2006. Thermo-mechanical response of nylon 101 under uniaxial and multi-axial loadings: Part I, Experimental results over wide ranges of temperatures and strain rates. International journal of plasticity 22 (8), 1506-1529.

Khan, A. S., Lopez-Pamies, O., Kazmi, R., 2006. Thermo-mechanical large deformation response and constitutive modeling of viscoelastic polymers over a wide range of strain rates and temperatures. International Journal of Plasticity 22 (4), 581-601.

Khan, F., Yeakle, C., Apr. 2011. Experimental investigation and modeling of nonmonotonic creep behavior in polymers. International Journal of Plasticity 27 (4), $512-521$.

URL http://www.sciencedirect.com/science/article/pii/S0749641910000975

Krairi, A., Doghri, I., 2014. A thermodynamically-based constitutive model for thermoplastic polymers coupling viscoelasticity, viscoplasticity and ductile damage. International Journal of Plasticity 60, 163-181.

Krempl, E., Khan, F., 2003. Rate (time)-dependent deformation behavior: an overview of some properties of metals and solid polymers. International Journal of Plasticity 19 (7), 1069-1095.

Lai, D., Yakimets, I., Guigon, M., Sep. 2005. A non-linear viscoelastic model developed for semi-crystalline polymer deformed at small strains with loading and unloading paths. Materials Science and Engineering: A 405 (1), 266-271.

URL http://www.sciencedirect.com/science/article/pii/S0921509305005629

Laiarinandrasana, L., Klinkova, O., Nguyen, F., Proudhon, H., Morgeneyer, T. F., Ludwig, W., Aug. 2016. Three dimensional quantification of anisotropic void evolution in deformed semi-crystalline polyamide 6. International Journal of Plasticity $83,19-36$.

URL https://linkinghub.elsevier.com/retrieve/pii/S0749641916300444

Lemaitre, J., 1992. A course on damage mechanics. Springer-Verlag Berlin.

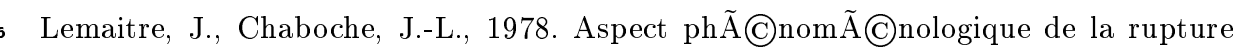
par endommagement. J Mec Appl 2 (3).

68 Lemaitre, J., Chaboche, J.-L., 1994. Mechanics of solid materials. Cambridge university press.

Li, H. X., Buckley, C. P., Apr. 2009. Evolution of strain localization in glassy polymers: A numerical study. International Journal of Solids and Structures 46 (7), 1607-1623.

URL http://www.sciencedirect.com/science/article/pii/S0020768308005039 
Lion, A., Johlitz, M., May 2016. A thermodynamic approach to model the caloric 774 properties of semicrystalline polymers. Continuum Mechanics and Thermodynamics 28 (3), 799-819.

Lion, A., Mittermeier, C., Johlitz, M., 2017. Heat capacities and volumetric changes in the glass transition range: a constitutive approach based on the standard linear solid. Continuum Mechanics and Thermodynamics 29 (5), 1061-1079.

Makradi, A., Ahzi, S., Gregory, R. V., Edie, D. D., Apr. 2005. A two-phase selfconsistent model for the deformation and phase transformation behavior of polymers above the glass transition temperature: application to PET. International Journal of Plasticity 21 (4), 741-758.

URL http://www.sciencedirect.com/science/article/pii/S0749641904000816

Maurel-Pantel, A., Baquet, E., Bikard, J., Billon, N., 2011. Coupled Thermo Mechan-

786

788

Maurel-Pantel, A., Baquet, E., Bikard, J., Bouvard, J. L., Billon, N., 2015. A thermomechanical large deformation constitutive model for polymers based on material network description: Application to a semi-crystalline polyamide 66. International Journal of Plasticity 67, 102-126.

URL http://www.sciencedirect.com/science/article/pii/S0749641914001983

Miled, B., Doghri, I., Delannay, L., 2011. Coupled viscoelastic-viscoplastic modeling of homogeneous and isotropic polymers: Numerical algorithm and analytical solutions. Computer Methods in Applied Mechanics and Engineering 200 (47), 3381-3394.

Nikolov, S., Doghri, I., 2000. A micro/macro constitutive model for the smalldeformation behavior of polyethylene. Polymer 41 (5), 1883-1891.

Nikolov, S., Doghri, I., Pierard, O., Zealouk, L., Goldberg, A., Nov. 2002. Multiscale constitutive modeling of the small deformations of semi-crystalline polymers. Journal of the Mechanics and Physics of Solids 50 (11), 2275-2302.

802 URL http://www.sciencedirect.com/science/article/pii/S0022509602000364

Ognedal, A. S., Clausen, A. H., Dahlen, A., Hopperstad, O. S., May 2014. Behavior of 
study. Mechanics of Materials 72, 94-108.

Praud, F., Chatzigeorgiou, G., Bikard, J., Meraghni, F., 2017. Phenomenological multi-mechanisms constitutive modelling for thermoplastic polymers, implicit implementation and experimental validation. Mechanics of Materials 114, 9-29.

Regrain, C., Laiarinandrasana, L., Toillon, S., Sa $\tilde{A}^{-}$, K., Jul. 2009. Multi-mechanism models for semi-crystalline polymer: Constitutive relations and finite element implementation. International Journal of Plasticity 25 (7), 1253-1279.

URL http://www.sciencedirect.com/science/article/pii/S0749641908001496

Reis, J. M. L., Pacheco, L. J., da Costa Mattos, H. S., 2013. Influence of the temperature and strain rate on the tensile behavior of post-consumer recycled high-density

816 polyethylene. Polymer Testing 32 (8), 1576-1581.

Schapery, R., jul 1967. Stress analysis of viscoelastic composite materials. Journal of Composite Materials 1 (3), 228-267.

Schapery, R. A., 1969. On the characterization of nonlinear viscoelastic materials. Polymer Engineering \& Science 9 (4), 295-310.

Shukla, R. S., Mertens, J., Senthilvelan, S., 2014. Hysteresis Heating of Polypropylene Based Composites. In: 5 th International \& 26 th All India Manufacturing Technology, Design and Research Conference (AIMTDR 2014). p. 6.

Simo, J., Hughes, T., 1998. Computational Inelasticity.

Sweeney, J., Collins, T. L. D., Coates, P. D., Unwin, A. P., Duckett, R. A., Ward, I. M., Mar. 2002. Application of a large deformation model to unstable tensile stretching of polyethylene. International Journal of Plasticity 18 (3), 399-414.

828 URL http://www.sciencedirect.com/science/article/pii/S0749641900001042

Sweeney, J., Ward, I. M., Jan. 1995. Rate dependent and network phenomena in the multiaxial drawing of poly(vinyl chloride). Polymer 36 (2), 299-308.

URL http://www.sciencedirect.com/science/article/pii/003238619591317Z

Uchida, M., Tada, N., Oct. 2013. Micro-, meso- to macroscopic modeling of deformation behavior of semi-crystalline polymer. International Journal of Plasticity

83449 (Supplement C), 164-184.

URL http://www.sciencedirect.com/science/article/pii/S0749641913000776 
Van Dommelen, J. v., Parks, D., Boyce, M., Brekelmans, W., Baaijens, F., 2003. Micromechanical modeling of the elasto-viscoplastic behavior of semi-crystalline poly-

838 mers. Journal of the Mechanics and Physics of Solids 51 (3), 519-541.

Wu, P., Van Der Giessen, E., 1993. On improved network models for rubber elasticity 840 and their applications to orientation hardening in glassy polymers. Journal of the Mechanics and Physics of Solids 41 (3), 427-456.

842 Wunderlich, B., Mar. 2003. Reversible crystallization and the rigid-amorphous phase in semicrystalline macromolecules. Progress in Polymer Science 28 (3), 383-450. URL http://www.sciencedirect.com/science/article/pii/S0079670002000850

Yu, C., Kang, G., Lu, F., Zhu, Y., Chen, K., 2016. Viscoelastic-viscoplastic cyclic deformation of polycarbonate polymer: experiment and constitutive model. Journal of Applied Mechanics 83 (4), 041002.

Zairi, F., Nait-Abdelaziz, M., Gloaguen, J.-M., Lefebvre, J.-M., 2008. Modelling of the elasto-viscoplastic damage behaviour of glassy polymers. International Journal of Plasticity 24 (6), 945-965.

Zhou, Y., Mallick, P. K., Dec. 2002. Effects of temperature and strain rate on the tensile behavior of unfilled and talc-filled polypropylene. Part I: Experiments. Polymer Engineering \& Science 42 (12), 2449-2460. 